\title{
On the Geometry and Mass of Static, Asymptotically AdS Spacetimes, and the Uniqueness of the AdS Soliton
}

\author{
G.J. Galloway ${ }^{a *}$, S. Surya ${ }^{b, c \dagger}$, E. Woolgar ${ }^{c, b \ddagger}$ \\ ${ }^{a}$ Dept. of Mathematics, University of Miami \\ Coral Gables, FL 33124, USA \\ ${ }^{b}$ Theoretical Physics Institute, Dept. of Physics \\ University of Alberta, Edmonton, AB, Canada T6G 2J1 \\ ${ }^{c}$ Dept. of Mathematical and Statistical Sciences \\ University of Alberta, Edmonton, AB, Canada T6G 2G1
}

\begin{abstract}
We prove two theorems, announced in hep-th/0108170 for static spacetimes that solve Einstein's equation with negative cosmological constant. The first is a general structure theorem for spacetimes obeying a certain convexity condition near infinity, analogous to the structure theorems of Cheeger and Gromoll for manifolds of non-negative Ricci curvature. For spacetimes with Ricci-flat conformal boundary, the convexity condition is associated with negative mass. The second theorem is a uniqueness theorem for the negative mass AdS soliton spacetime. This result lends support to the new positive mass conjecture due to Horowitz and Myers which states that the unique lowest mass solution which asymptotes to the AdS soliton is the soliton itself. This conjecture was motivated by a nonsupersymmetric version of the AdS/CFT correspondence. Our results add to the growing body of rigorous mathematical results inspired by the AdS/CFT correspondence conjecture. Our techniques exploit a special geometric feature which the universal cover of the soliton spacetime shares with familiar "ground state" spacetimes such as Minkowski spacetime, namely, the presence of a null line, or complete achronal null geodesic, and the totally geodesic null hypersurface that it determines. En route, we provide an analysis of the boundary data at conformal infinity for the Lorentzian signature static Einstein equations, in the spirit of the Fefferman-Graham analysis for the Riemannian signature case. This leads us to generalize to arbitrary dimension a mass definition for static asymptotically AdS spacetimes given by Chruściel and Simon. We prove equivalence of this mass definition with those of Ashtekar-Magnon and Hawking-Horowitz.
\end{abstract}

*galloway@math.miami.edu

†ssurya@phys.ualberta.ca

${ }^{\ddagger}$ ewoolgar@math.ualberta.ca 


\section{Introduction}

There has been considerable interest in the last few years in the AdS/CFT correspondence first proposed in [30, 40, 19]. This correspondence equates the string partition function on an anti-de Sitter (AdS) background to that of a conformal field theory on the AdS boundary-at-infinity (the Penrose conformal boundary, scri), and is an explicit realisation of the holographic principle suggested in [38, 36]. In particular the large $N$ limit of the conformal field theory corresponds to the low energy limit of string theory, i.e., classical supergravity. In this limit, classical properties of a spacetime have definite interpretations in the gauge theory (see [27] for example).

Of particular interest is the question of what the positivity of gravitational energy means in the conformal field theory. Related to this is the role of "ground" states, namely, the lowest energy configurations classically allowed, satisfying certain physically reasonable conditions. In asymptotically flat space, the celebrated positive energy theorem 35, 39. tells us that Minkowski space is the unique lowest energy solution provided that the local energy density is non-negative and that there are no naked singularities. In the presence of a negative cosmological constant, the analogous ground state is AdS spacetime. Both Minkowski spacetime and AdS are regular, static, supersymmetric and of constant curvature. For each of these familiar ground states there is an associated uniqueness theorem [29, 5, 9, 8] showing that the spacetime is unique in the class of regular, stationary, vacuum solutions when the appropriate fall-off conditions are satisfied.

The standard conditions for an $(n+1)$-dimensional spacetime to be asymptotically AdS include a specification of the topology of the $n$-dimensional conformal boundary, i.e., that it be $S^{n-1} \times \mathbb{R}[4$. When this topological restriction is relaxed, black hole spacetimes which are asymptotically locally AdS and which have nonspherical horizons are known to exist ${ }^{1}$ (for a review, see 31]). The timelike conformal infinities of these black holes have nonspherical cross-sections [15]. However, a conformal boundary with cross-sections of nonspherical topology cannot also serve as the conformal boundary of a nonsingular locally AdS spacetime. Thus, AdS or its topological modifications cannot be appropriate ground states for such black hole spacetimes.

Thus we are led to entertain the somewhat radical proposition that ground states for spacetimes with nonspherical scri may not be conformally flat, and thus not massless according to familiar mass formulae such as Ashtekar-Magnon [4]. For the case of toroidal scri, a candidate ground state has been proposed by Horowitz and Myers 25, which they dubbed the "AdS soliton". In $n+1 \geq 4$ spacetime dimensions, ${ }^{2}$ it is a globally static Einstein spacetime with cosmological constant $\Lambda<0$ and has the form

$$
d s^{2}=-r^{2} d t^{2}+\frac{d r^{2}}{V(r)}+V(r) d \phi^{2}+r^{2} \sum_{i=1}^{n-2}\left(d \theta^{i}\right)^{2}
$$

Here, $V(r)=\frac{r^{2}}{\ell^{2}}\left(1-\frac{r_{0}^{n}}{r^{n}}\right)$, with $\ell^{2}=\frac{n(n-1)}{-2 \Lambda}$ and $r_{0}$ is a constant. The solution is nonsingular

\footnotetext{
${ }^{1}$ In what follows, the term locally Anti-de Sitter spacetime means "constant negative curvature spacetime", while Anti-de Sitter (AdS) spacetime refers to the unique, geodesically complete, simply connected, constant negative curvature spacetime. An asymptotically AdS spacetime has spherical scri, but one that is merely asymptotically locally AdS need not have spherical scri.

${ }^{2}$ In $2+1$ spacetime dimensions, this soliton is identical to 3 -dimensional AdS.
} 
provided $\phi$ is periodic with period $\beta_{0}=\frac{4 \pi \ell^{2}}{n r_{0}}$. The periods of the $\theta^{i}$ are arbitrary. The soliton is asymptotically locally Anti-de Sitter with boundary-at-infinity (scri) foliated by spacelike $(n-1)$-tori. Spacetime itself, when conformally completed, has constant time slices which are topologically the product of an $(n-2)$-torus and a disk (in $3+1$ dimensions, it is therefore a solid torus). Moreover, the soliton is neither of constant curvature nor supersymmetric, but it is known to have minimal energy under small metric perturbations 25. 10.

Curiously, the AdS soliton has negative mass relative to the natural choice that associates the zero of energy with conformal flatness. The mass $E_{0}$ depends on the period $\beta_{0}$ of the $\phi$ coordinate according to

$$
E_{0}=\frac{-V_{n-2} \ell^{n-1}}{16 \pi G_{n+1} \beta_{0}^{n-1}}\left(\frac{4 \pi}{n}\right)^{n},
$$

where $V_{n-2}$ is the product of the periods $\beta_{i}$ of the $\theta^{i}$, these being arbitrary, and $G_{n+1}$ is Newton's constant in the $(n+1)$-dimensional spacetime. A simple argument may suggest that by rescaling the parameters of a negative mass spacetime, one could get a spacetime of even lower mass. However, for the AdS soliton, the proposed rescaling is an isometry, and does not change the mass [16]. ${ }^{3}$ Thus, a negative mass ground state, in this case, need not be a contradiction after all. Page 32] has introduced a scale-invariant mass

$$
\epsilon:=E_{0} V_{n-1}:=E_{0} V_{n-2} \beta_{0}=-C\left(\frac{<\beta>}{\beta_{0}}\right)^{n},
$$

where $C$ is a constant that depends on $n$ but is invariant under rescalings of the periods, and $\langle\beta\rangle:=\left(\beta_{0} \beta_{1} \ldots \beta_{n-2}\right)^{1 /(n-1)}$ is the geometric mean of these periods, including $\beta_{0}$.

Horowitz and Myers found that the negative mass of the AdS soliton has a natural interpretation as the Casimir energy of a nonsupersymmetric gauge theory on the conformal boundary. If a nonsupersymmetric version of the AdS/CFT conjecture is to hold, as is generally hoped, then this would indicate that the soliton is the lowest energy solution with these boundary conditions. This led them to postulate a new positive energy conjecture, that the soliton is the unique lowest mass solution for all spacetimes in its asymptotic class. The validity of this conjecture is thus an important test of the nonsupersymmetric version of the AdS/CFT correspondence. ${ }^{4}$

A preliminary indication that the soliton is the appropriate ground state comes from a semi-classical thermodynamic analysis of Ricci flat black holes in the background of the AdS soliton [37. An examination of the thermodynamics of the spherical AdS black hole showed that there is a phase transition that takes place between the black hole and the appropriate ground state, namely AdS spacetime [22]. This phase transition was interpreted as a confinement/deconfinement transition in the associated large $N$ gauge theory on the boundary [40]. Taking a cue from this, it was shown that a phase transition

\footnotetext{
${ }^{3}$ The black hole solutions of Lemos [28] are also mutually isometric under this scaling, and a similar scaling works for the 5-dimensional nilgeometry and solvegeometry black holes of [6].

${ }^{4}$ Page (32] points out that, given $n-1$ distinct positive numbers $b_{\mu}$, there are $n-1$ distinct solitons for which these numbers serve as boundary data specifying the periods of the coordinates, depending on which one of them is chosen to equal the period $\beta_{0}$ of $\phi$, and that the invariant energy can be minimized only if $\beta_{0}=\operatorname{Min}_{\mu}\left\{b_{\mu}\right\}$.
} 
also occurs between the toroidal black hole and the AdS soliton, and can also be interpreted as a confinement/deconfinement transition in the boundary field theory, in much the same way [37]. Indeed the choice of ground state is crucial to see such a phase transition. Earlier analysis which identified the locally AdS spacetime with toroidal boundary (which is singular) as the ground state did not give rise to such a phase transition.

In support of the Horowitz-Myers new positive energy conjecture, we prove a uniqueness theorem for the AdS soliton, singling it out as the only suitable ground state in a large class of negative mass spacetimes obeying certain boundary conditions. Our results are similar in spirit to those of [29, 5], but relate to asymptotically locally AdS spacetime with Ricci flat scri. The key elements of the proof are, briefly, (a) the use of negative mass and certain asymptotic conditions (related to the convexity of constant lapse hypersurfaces near scri) to establish the existence of null lines in the universal covering spacetime and (b) the construction, using the null splitting theorem due to Galloway ([14], quoted below as Theorem I.1), of a foliation of spacetime based on totally geodesic null hypersurfaces.

A null line in spacetime is an inextendible null geodesic which is globally achronal, i.e., for which no two points can be joined by a timelike curve. (Hence, each segment of a null line is maximal with respect to the Lorentzian distance function.) Arguments involving null lines have arisen in numerous situations, such as the Hawking-Penrose singularity theorems [20], results on topological censorship ([15], and references cited therein), and the Penrose-Sorkin-Woolgar approach to the positive mass theorem [33, 41] and related results on gravitational time delay [18. It will be convenient for the purposes of the present paper to require null lines to be not only inextendible, but geodesically complete.

For the reader's convenience, we quote here the null splitting theorem:

Theorem I.1 (Galloway [14]). If a null geodesically complete spacetime obeys $R_{a b} X^{a} X^{b}$ $\geq 0$ for all null vectors $X^{a}$ and also contains a null line $\eta$, then $\eta$ lies in a smooth, achronal, edgeless, totally geodesic null hypersurface $\mathcal{H}$.

Remark I.2. (a) The spacetimes considered herein will be vacuum (with negative cosmological constant), so they obey $R_{a b} X^{a} X^{b}=0$ for all null vectors $X^{a}$. (b) Because $\mathcal{H}$ is totally geodesic, the tangent vector field $n^{a}$ to the null geodesic generators of $\mathcal{H}$, when suitably scaled, is a parallel vector field along $\mathcal{H}, X^{a} \nabla_{a} n^{b}=0$ for all tangent vectors $X$ to $\mathcal{H}$. We will make use of this fact in Section III.

In Section III we consider a boundary value problem for asymptotically locally AdS spacetimes with Ricci flat conformal boundary. We draw on a formalism of Chruściel and Simon [9] for static, asymptotically AdS solutions, but while they restrict to 4 spacetime dimensions, we work in $n+1$ spacetime dimensions. To discuss boundary conditions, and in particular to relate the sign of the mass to data on the Penrose conformal boundary, we express the extrinsic geometry of hypersurfaces approaching scri as an expansion in a certain coordinate distance from scri, and use this to expand the scalar curvature of the conformal metric on constant time slices. Similar analyses appear in [23, 13, 24, 9, 2, We find that in the static spacetime setting with Ricci flat boundary a power series expansion suffices for both even and odd dimension $n$, i.e., no log terms arise in the expansion. The only free data is the induced boundary conformal metric and its $n^{\text {th }}$ "radial" derivative, which we relate to the mass. We find it convenient to use the Chruściel-Simon mass definition, generalized to arbitrary dimension. In Section II.3, we prove equivalence of this 
mass and the Ashtekar-Magnon mass. A proof of equivalence to the Hawking-Horowitz mass is consigned to Appendix A. Given these equivalences, then equivalence to other common mass definitions (esp. that of Abbott and Deser [1]) follows from existing results 21].

In Section III we present the first of our main theorems, a structure theorem. Here we show, roughly speaking, that given a certain convexity condition near infinity, then in the universal covering space of a constant time slice in the conformal spacetime, the noncompact directions split off from the compact directions, and are flat. This result is analogous to a structure theorem of Cheeger and Gromoll [7. The proof works by using our asymptotic conditions to establish the presence of a line in the universal cover. This line can be lifted to a null line in the universal cover of the physical spacetime; this is proved in Appendix B. By the null splitting theorem I.1, spacetimes with null lines have a special geometry, and this yields our structure result. By further imposing certain topological restrictions, we are then led to a uniqueness theorem for the soliton which we prove in Section [IV] The assumption of negative mass in the uniqueness theorem is used, via the results of Section II, to show that the aforementioned convexity condition holds in the mean, thereby permitting a weakening of the convexity condition (cf. the discussion of Conditions (C) and (S) in Section III.1). We very briefly mention some extensions of our results in Section $\nabla$.

Of related interest, Anderson [2] has proved uniqueness for 4-dimensional hyperbolic (thus, Riemannian) metrics, provided certain coefficients in the expansion of the conformal metric are fixed on the conformal boundary. Also, Kiem and Park [26] have shown uniqueness of the soliton but only under very strong assumptions, among which, for example, is the structure theorem that we will prove in Section III.

The main theorems proved in Sections III and IV were announced in [16]. Herein, we provide explicit, detailed proofs and associated analyses and lay a basis for the further future work [17] briefly touched upon in Section $\nabla$. Throughout, the spacetime dimension is $n+1$.

\section{The Boundary Value Problem}

The static Einstein equations in the asymptotically locally de Sitter setting form a highly nonlinear elliptic, asymptotically degenerate, system of equations, and it is not clear $a$ priori that the AdS soliton would be a unique solution even if all the necessary boundary data were specified. We write out the field equations with respect to the physical metric and also with respect to a relevant conformally related metric in Section II.1. In Section II.2, we find that the free data on the (Ricci flat) conformal boundary are the induced metric and its normal derivatives of order $n$; the latter also determine the mass of the spacetime. It is convenient to use a mass definition based on that of Chruściel and Simon [9], so in Section II.3 we prove equivalence of this mass to the familiar Ashtekar-Magnon mass. A feature of our uniqueness theorem is that we will not need to specify all the free data on the conformal boundary to obtain uniqueness. Apart from the induced boundary metric, we will specify only that the sign of the mass is negative, though we must pay a price by requiring further topological assumptions and an assumption on the extrinsic geometry of constant lapse surfaces near infinity (cf. Section III.1). 


\section{II.1 The Field Equations}

We consider $(n+1)$-dimensional, $n \geq 2$, static spacetimes $(\mathcal{M}, g)$,

$$
\begin{aligned}
\mathcal{M} & =\mathbb{R} \times \Sigma, \\
g & =-N^{2} d t^{2} \oplus h,
\end{aligned}
$$

where $h$ is the induced metric on $\Sigma$ and $N$ is the lapse, such that the triple $(\Sigma, h, N)$ is $C^{k}$ $(k \geq 2)$ conformally compactifiable. Thus, $\Sigma$ is the interior of a smooth compact manifold with boundary $\widetilde{\Sigma}=\Sigma \cup \partial \widetilde{\Sigma}$ such that

(a) $N^{-1}$ extends to a $C^{k}$ function $\widetilde{N}$ on $\widetilde{\Sigma}$, with $\left.\widetilde{N}\right|_{\partial \widetilde{\Sigma}}=0$ and $\left.d \widetilde{N}\right|_{\partial \widetilde{\Sigma}} \neq 0$ pointwise, and

(b) $N^{-2} h$ extends to a $C^{k}$ Riemannian metric $\widetilde{h}$ known as the Fermat (or optical) metric on $\widetilde{\Sigma}$.

The definition of conformally compactifiable given here precludes the existence of internal boundaries, such black hole boundaries. While this preclusion is not needed for the asymptotic analysis presented in this section, it is used in Sections III and IV, which are concerned with properties of globally static spacetimes. The case of black holes will be dealt with in a forthcoming paper [17.

The triplet $(\Sigma, h, N)$ obeys the static vacuum field equations

$$
\begin{aligned}
R_{a b} & =\frac{1}{N} \nabla_{a} \nabla_{b} N+\frac{2 \Lambda}{n-1} h_{a b}, \\
\Delta N & =-\frac{2 \Lambda}{n-1} N,
\end{aligned}
$$

where $\nabla_{a}$ and $R_{a b}$ are respectively the covariant derivative and Ricci tensor on $(\Sigma, h)$, and $\Lambda<0$ is the cosmological constant. These spacetimes are asymptotically constant negative curvature.

These equations can be rewritten in terms of the Fermat metric $\widetilde{h}$ and associated $\widetilde{\nabla}_{a}$ and $\widetilde{R}_{a b}$ as

$$
\begin{aligned}
\widetilde{R}_{a b} & =\frac{-(n-1)}{\widetilde{N}} \widetilde{\nabla}_{a} \widetilde{\nabla}_{b} \widetilde{N}, \\
\widetilde{N} \widetilde{\Delta} \widetilde{N} & =\left(\frac{2 \Lambda}{n-1}+n \widetilde{W}\right),
\end{aligned}
$$

where

$$
\widetilde{W}:=\widetilde{h}^{a b} \widetilde{\nabla}_{a} \widetilde{N} \widetilde{\nabla}_{b} \widetilde{N}=\frac{1}{N^{2}} h^{a b} \nabla_{a} N \nabla_{b} N .
$$

A useful identity is obtained by taking the trace of (II.1.4) and combining this with (II.1.5):

$$
\widetilde{N}^{2} \widetilde{R}+2 \Lambda+n(n-1) \widetilde{W}=0 .
$$

Solving for $\Lambda$ and reinserting into (II.1.5), we obtain

$$
\left(\widetilde{\Delta}+\frac{\widetilde{R}}{n-1}\right) \widetilde{N}=0
$$


We will sometimes state results in terms of the triple $(\Sigma, h, N)$, but because we assume conformal compactifiability, we will often work with $(\widetilde{\Sigma}, \widetilde{h}, \widetilde{N})$, and therefore we often work with the system (II.1.4 (I.1.6) rather than (II.1.2 (I.1.3).

We end the section with an identity that will be of use in our discussion of mass and boundary conditions. We define $x:=\widetilde{N}=1 / N$ and let $\partial / \partial x$ be the vector dual to $d x$ under the isomorphism defined by the Fermat metric $\widetilde{h}_{a b}$. If we then Fermat normalize this dual vector, we obtain the vector

$$
\widetilde{n}^{a}:=\frac{-1}{\sqrt{\widehat{W}}}\left(\frac{\partial}{\partial x}\right)^{a}=\frac{-1}{\sqrt{\widehat{W}}} \widetilde{h}^{a b} \widetilde{\nabla}_{b} \widetilde{N}
$$

Note that we have also reversed the sense of the vector by introducing a minus sign. This is for convenience in the next section, where we will use this formula in the case where $\widetilde{n}^{a}$ will be normal to scri, and we will want it to be the outward directed normal, pointing in the direction of decreasing $x$. Now from the definition (II.1.6) we have

$$
\begin{aligned}
\frac{\partial \widetilde{W}}{\partial x}=\frac{-1}{\sqrt{\widetilde{W}}} \widetilde{n}^{a} \widetilde{\nabla}_{a} \widetilde{W} & =\frac{-2}{\sqrt{\widetilde{W}}}\left(\widetilde{h}^{a b} \widetilde{\nabla}_{a} x\right)\left(\widetilde{n}^{c} \widetilde{\nabla}_{c} \widetilde{\nabla}_{b} x\right) \\
& =\frac{-2 x}{(n-1)} \widetilde{R}_{a b} \widetilde{n}^{a} \widetilde{n}^{b}
\end{aligned}
$$

where in the last step we used (II.1.4). If we now differentiate (II.1.7) with respect to $x=\widetilde{N}$, use the results in the left-hand side of (II.1.10), and rearrange terms, we obtain

$$
\widetilde{R}_{a b} \widetilde{n}^{a} \widetilde{n}^{b}-\frac{1}{n} \widetilde{R}=\frac{x}{2 n} \frac{\partial \widetilde{R}}{\partial x}
$$

\section{II.2 The Boundary Conditions}

The solution of the field equations on $\Sigma$ of course will not be unique unless we specify some boundary data on $\partial \widetilde{\Sigma}$, which here is the hypersurface $x:=\widetilde{N}=0$. The data we wish to specify are the induced metric on $\partial \widetilde{\Sigma}$ and the sign of the mass of spacetime. The latter is related to the $n^{\text {th }}$ order $x$-derivatives of the Fermat metric coefficients at $x=0$.

Many similar analyses have appeared in the literature, among them [23, 13, 24, 9, 2. These analyses usually focus on the issue of whether the vacuum Einstein equations admits a formal power series solution centred at conformal infinity or whether the power series must be supplemented by log terms. Typically, these analyses deal with the full vacuum Einstein equations, either with zero [13] or negative 24] cosmological constant. We will deal with the static (thus, Lorentzian signature) Einstein equations in dimension $n+1$, and will focus on the case of Ricci-flat conformal boundary since this case includes the AdS soliton. The assumption of a timelike Killing field yields a more restrictive system of equations than the general system with no symmetries in $n+1$ dimensions (that it is not equivalent to the $n$-dimensional system is evident, cf. (II.1.2) below). We find formal power series solutions for all $n$, in agreement with the results of [24] in dimensions 2, 4, and 6 .

We show below that the first $n-1 x$-derivatives of the Fermat metric components at $x=0$ vanish if this metric is assumed to be of class $C^{n}$ there. From what follows, it can 
be seen that if the order $n x$-derivatives of the Fermat metric components are supplied at $x=0$, then all higher $x$-derivatives are determined there, up to one order below that at which differentiability fails. This behaviour does not depend on whether $n$ is even or odd. When the Fermat metric is of class $C^{\infty}$ at $x=0$, this technique yields a formal power series solution of the field equations at $x=0$. Here we do not assume $C^{\infty}$, nor do we concern ourselves with convergence of the power series, preferring instead to obtain our uniqueness theorem by geometric techniques. An important ingredient of these techniques will be Corollary II.2.3, which relates the sign of the mass aspect (Definition II.3.2) to the mean curvature of constant lapse surfaces near infinity.

Near the boundary $x=0$, we may introduce coordinates $x^{1}=x, x^{2}, \ldots, x^{n}$ so that the metric $\widetilde{h}$ takes the form,

$$
\widetilde{h}=\frac{d x^{2}}{\widetilde{W}}+\widetilde{b}_{\alpha \beta} d x^{\alpha} d x^{\beta}
$$

where $\widetilde{b}_{\alpha \beta}=\widetilde{b}_{\alpha \beta}\left(x, x^{\gamma}\right)$ is the induced metric on the constant $x$ slice $V_{x} \approx \partial \widetilde{\Sigma}$ (here and throughout this subsection, Greek indices run from 2 to $n$ ). The second fundamental form $\widetilde{H}_{\alpha \beta}=\widetilde{H}_{\alpha \beta}(x)$ of $V_{x}$ is defined as $\widetilde{H}_{\alpha \beta}=\widetilde{\nabla}_{\alpha} \widetilde{n}_{\beta}$, from which it follows that

$$
\widetilde{H}_{\alpha \beta}=\frac{-1}{2 \psi} \partial_{x} \widetilde{b}_{\alpha \beta}
$$

where $\psi:=\widetilde{W}^{-1 / 2}$.

By taking the projections of the field equation (II.1.4) tangent and normal to each $V_{x}$, we obtain

$$
\begin{aligned}
& \widetilde{R}_{\alpha \beta}=\frac{(n-1)}{x \psi} \widetilde{H}_{\alpha \beta}, \\
& \widetilde{R}_{x x}=\frac{(n-1)}{x \psi} \partial_{x} \psi .
\end{aligned}
$$

We may use the standard expression for the Ricci curvature in terms of Christoffel symbols to expand the left-hand sides of equations (II.2.3 II.2.4). Doing so, we obtain

$$
\begin{aligned}
\widetilde{H}_{\alpha \beta} & =\frac{x}{n-1}\left(\partial_{x} \widetilde{H}_{\alpha \beta}+\left[2 \widetilde{H}_{\alpha}^{\gamma} \widetilde{H}_{\beta \gamma}-\widetilde{H} \widetilde{H}_{\alpha \beta}+\mathcal{R}_{\alpha \beta}-D_{\alpha} D_{\beta}\right] \psi\right), \\
\partial_{x} \psi(x) & =\frac{x}{n-1} \psi^{2}\left(\partial_{x} \widetilde{H}-\left[D^{2}+\widetilde{H}_{\alpha \beta} \widetilde{H}^{\alpha \beta}\right] \psi\right)
\end{aligned}
$$

where $D_{\alpha}$ is the Levi-Cevita connection of the induced metric on $V_{x}$ and $\mathcal{R}_{\alpha \beta}$ is its Ricci

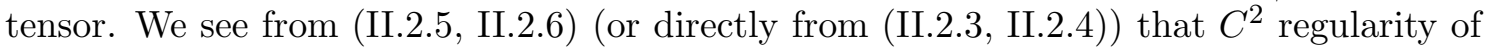
the Fermat metric at $x=0$ requires that

$$
\widetilde{H}_{\alpha \beta}(0)=\partial_{x} \psi(0)=0
$$

If the terms in (II.2.5 【I.2.6) are $k-1$ times differentiable then, taking $k \geq 2$, we may apply $\partial_{x}^{k-1}:=\partial^{k-1} / \partial x^{k-1}$ to these equations to obtain, for $n \neq k$, the following 
expressions:

$$
\begin{aligned}
\partial_{x}^{k-1} \widetilde{H}_{\alpha \beta}= & \frac{x}{(n-k)} \partial_{x}^{k-1}\left(\partial_{x} \widetilde{H}_{\alpha \beta}+\left[2 \widetilde{H}_{\alpha}^{\gamma} \widetilde{H}_{\beta \gamma}-\widetilde{H} \widetilde{H}_{\alpha \beta}+\mathcal{R}_{\alpha \beta}-D_{\alpha} D_{\beta}\right] \psi\right) \\
& +\frac{(k-1)}{(n-k)} \partial_{x}^{k-2}\left(\left[2 \widetilde{H}_{\alpha}^{\gamma} \widetilde{H}_{\beta \gamma}-\widetilde{H} \widetilde{H}_{\alpha \beta}+\mathcal{R}_{\alpha \beta}-D_{\alpha} D_{\beta}\right] \psi\right), \\
\partial_{x}^{k} \psi= & \frac{x}{(n-1)} \partial_{x}^{k-1}\left(\psi^{2} \partial_{x} \widetilde{H}-\psi^{2}\left[D^{2}+\widetilde{H}_{\alpha \beta} \widetilde{H}^{\alpha \beta}\right] \psi\right) \\
& +\frac{(k-1)}{(n-1)} \partial_{x}^{k-2}\left(\psi^{2} \partial_{x} \widetilde{H}-\psi^{2}\left[D^{2}+\widetilde{H}_{\alpha \beta} \widetilde{H}^{\alpha \beta}\right] \psi\right)
\end{aligned}
$$

Proposition II.2.1. Let $(\Sigma, h, N)$ be $C^{m+1}$ conformally compactifiable. We assume the conformal boundary hypersurface $x=0$ to be Ricci flat. (i) If $m<n:=\operatorname{dim} \Sigma$, the first $m x$-derivatives of $\psi$ and of $\widetilde{b}_{\alpha \beta}$ (equivalently, from (II.2.2),$\widetilde{H}_{\alpha \beta}(x)$ and its first $m-1$ $x$-derivatives) vanish at $x=0$. (ii) If $m \geq n$, the first $n-1 x$-derivatives of $\psi$ and $\widetilde{b}_{\alpha \beta}(x)$ vanish at $x=0$ and the remaining $x$-derivatives up to order $m-1$ inclusive are completely determined by $\widetilde{b}_{\alpha \beta}(0)$ and $\widetilde{b}_{\alpha \beta}^{(n)}(0)$ (equivalently, $\widetilde{H}_{\alpha \beta}^{(n-1)}(0)$ ).

Proof. By assumption, the Fermat metric is $C^{m+1}$ differentiable, which is equivalent to $C^{m+1}$ differentiability of $\psi$ and $\widetilde{b}_{\alpha \beta}$, implying $C^{m}$ differentiability of $\widetilde{H}_{\alpha \beta}$. The idea of the proof is simple. Equations (II.2.8, ஹ.2.9) are singular at $x=0$, but we can use $C^{m+1}$ regularity to control the behaviour of the highest derivatives on the right-hand sides. We can then eliminate the highest derivatives in these equations by setting $x=0$. The derivatives remaining on the right are then of lower order than those on the left, so we can proceed by induction.

To begin, observe that every term on the right-hand side of (II.2.9) can be written in terms of $\widetilde{b}_{\alpha \beta}$, its inverse, its first $k+1$ derivatives, and $\psi$ and its first $k+1$ derivatives. Thus, for $k \leq m$ we can use $C^{m+1}$ regularity to set $x=0$ in (II.2.9), thereby eliminating the highest derivatives and obtaining

$$
\begin{aligned}
\left.\partial_{x}^{k} \psi\right|_{0} & =\left.\frac{(k-1)}{(n-1)} \partial_{x}^{k-2}\left(\psi^{2} \partial_{x} \widetilde{H}-\psi^{2}\left[D^{2}+\widetilde{H}_{\alpha \beta} \widetilde{H}^{\alpha \beta}\right] \psi\right)\right|_{0} \\
= & : F_{(k)}\left(\widetilde{b}_{\alpha \beta}(0), \widetilde{H}_{\alpha \beta}(0), \ldots, \widetilde{H}_{\alpha \beta}^{(k-1)}(0), \psi(0), \ldots, \psi^{(k-2)}(0)\right),
\end{aligned}
$$

where the function $F_{(k)}$ is defined for $k \geq 2$, a superscript $p$ in parentheses denotes the $x$-derivative of order $p$, and the subscript 0 denotes evaluation at $x=0$. Note that $F_{(k)}$ can depend on tangential derivatives of its arguments (through the $D^{2} \psi$ term), although our notation does not make that explicit.

We want only order $k-1$ derivatives in $\widetilde{b}_{\alpha \beta}$ and $\psi$ in $F_{(k)}$, but the appearance of $\widetilde{H}_{\alpha \beta}^{(k-1)}$ prevents this. We can, however, express $\widetilde{H}_{\alpha \beta}^{(k-1)}$ in terms of lower order derivatives by using equation (I.2.8). Now every term in (II.2.8) can be written as a combination of $\widetilde{b}_{\alpha \beta}(x)$, its inverse, its derivatives, and $\psi$ and its derivatives, with the highest order derivatives appearing on the right-hand side, both of $\widetilde{b}_{\alpha \beta}$ and of $\psi$, being of order $k+1$. Therefore, provided $k \leq m$ and $k \neq n$, we can take $x=0$ in (II.2.8), again eliminating the 
highest derivatives. We obtain

$$
\begin{aligned}
\left.\partial_{x}^{k-1} \widetilde{H}_{\alpha \beta}\right|_{0} & =\left.\frac{(k-1)}{(n-k)} \partial_{x}^{k-2}\left(\left[2 \widetilde{H}_{\alpha}^{\gamma} \widetilde{H}_{\beta \gamma}-\widetilde{H} \widetilde{H}_{\alpha \beta}+\mathcal{R}_{\alpha \beta}-D_{\alpha} D_{\beta}\right] \psi\right)\right|_{0} \\
& =: G_{(k) \alpha \beta}\left(\widetilde{b}_{\alpha \beta}(0), \widetilde{H}_{\alpha \beta}(0), \ldots, \widetilde{H}_{\alpha \beta}^{(k-2)}(0), \psi(0), \ldots, \psi^{(k-2)}(0)\right),
\end{aligned}
$$

where the function $G_{(k) \alpha \beta}$ is defined for $k \geq 2$ and $k \neq n$. Thus, beginning at $k=2$ and excepting $k=n$, the system comprised of (II.2.11) and (II.2.13) expresses the order $k x$-derivatives of $\psi$ and $\widetilde{b}_{\alpha \beta}$ (order $k-1 x$-derivatives of $\widetilde{H}_{\alpha \beta}$ ) at $x=0$ in terms of the lower order $x$-derivatives at $x=0$.

We may solve these equations iteratively, beginning with $k=2$. To start the iteration, we must supply the data $\psi(0), \partial_{x} \psi(0), \widetilde{b}_{\alpha \beta}(0)$, and $\partial_{x} \widetilde{b}_{\alpha b}(0)$ or equivalently $\widetilde{H}_{\alpha \beta}(0)$. However, equation (II.2.7) fixes $\widetilde{H}_{\alpha \beta}(0)=\partial_{x} \psi(0)=0$, while $\psi(0)$ is determined by (II.1.7) and the definition $\widetilde{W}=1 / \psi^{2}$ to be

$$
\psi(0)=\sqrt{\frac{n(n-1)}{-2 \Lambda}} \equiv \ell
$$

The iteration proceeds until $k=n$, at which point it fails to assign a value to $\widetilde{H}_{\alpha \beta}^{(n-1)}(0)$ or equivalently to $\widetilde{b}_{\alpha \beta}^{(n)}(0)$. If a value for this quantity is assigned by fiat, the iteration can again proceed until the limit imposed by the assumed differentiability class of the Fermat metric is reached. Thus, the free data are $\widetilde{b}_{\alpha \beta}(0)$ and, if $k \geq n, b_{\alpha \beta}^{(n)}(0)$ (equivalently, $\left.\widetilde{H}_{\alpha \beta}^{(n-1)}(0)\right)$ as well.

Finally, since $\psi(0)=\ell$ is constant on the $x=0$ surface and since we assume $\mathcal{R}_{\alpha \beta}(0)=$ 0 , then one can see by applying the Leibniz rule in the definitions of $F_{(k)}$ and $G_{(k) \alpha \beta}$ that

$$
F_{(k)}\left(\widetilde{b}_{\alpha \beta}(0), 0, \ldots, 0, \psi(0), 0, \ldots, 0\right)=G_{(k) \alpha \beta}\left(\widetilde{b}_{\alpha \beta}(0), 0, \ldots, 0, \psi(0), 0, \ldots, 0\right)=0 .
$$

Thus, if all derivatives of $\widetilde{b}_{\alpha \beta}$ and $\psi$ below order $k$ vanish at $x=0$, then so do the order $k$ derivatives, unless $k=n$.

Corollary II.2.2. If $(\Sigma, h, N)$ is $C^{n+1}$ conformally compactifiable with Ricci flat conformal boundary $x=0$, where $n:=\operatorname{dim} \Sigma \geq 3$, then the Fermat scalar curvature $\widetilde{R}$ and its first $n-3 x$-derivatives vanish at $x=0$.

Proof. For $1<k<n$, the definition $\psi=1 / \sqrt{\widetilde{W}}$ yields

$$
\partial_{x}^{k} \widetilde{W}=-2 \frac{\partial_{x}^{k} \psi}{\psi^{3}}+\ldots+(-1)^{k}(k+1) ! \frac{\left(\partial_{x} \psi\right)^{k}}{\psi^{k+2}}
$$

where the dots represent $k-2$ terms, all containing $x$-derivatives of $\psi$ of order $<k$. Then Proposition II.2.1 implies the vanishing of the right-hand side of (II.2.16) at $x=0$. But, differentiating (II.1.7) $k \leq n$ times and setting $x=0$, we obtain

$$
\left.\partial_{x}^{k} \widetilde{W}\right|_{0}=\frac{-k(k-1)}{n(n-1)} \widetilde{R}^{(k-2)}(0)
$$


for $k \geq 2$. Since we have just shown that the left-hand side of this expression vanishes for $k<n$, so does the right-hand side, and so the first $n-3 x$-derivatives of $\widetilde{R}$ vanish at $x=0$ as claimed.

From (11.2.17), (11.2.16), and (11.2.11), we obtain

$$
\widetilde{R}^{(n-2)}(0)=\frac{2}{\psi^{3}(0)} F_{n}\left(\widetilde{b}_{\alpha \beta}(0), 0, \ldots, 0, \widetilde{H}_{\alpha \beta}^{(n-1)}(0), \psi(0), 0, \ldots, 0\right)
$$

Thus, $\widetilde{R}^{(n-2)}(0)$ and $\widetilde{H}_{\alpha \beta}^{(n-1)}(0)$ are related, so we may regard $\widetilde{R}^{(n-2)}(0)$ as part of the free data. We will soon see that $\widetilde{R}^{(n-2)}(0)$ encodes the mass, but first we determine the exact relation between it and $\widetilde{H}_{\alpha \beta}^{(n-1)}(0)$ :

Corollary II.2.3. If $(\Sigma, h, N)$ is $C^{n+1}$ conformally compactifiable with Ricci flat conformal boundary $x=0$, then the Fermat mean curvature of constant lapse surfaces near $x=0$ obeys

$$
\widetilde{H}(x)=\frac{\ell}{2} \frac{x^{n-1}}{(n-1) !} \widetilde{R}^{(n-2)}(0)+\mathcal{O}\left(x^{n}\right)
$$

Proof. If we contract (II.2.3) with $\widetilde{b}^{\alpha \beta}$, we obtain

$$
\widetilde{R}_{a b} \widetilde{n}^{a} \widetilde{n}^{b}-\widetilde{R}=-(n-1) \frac{\widetilde{H}}{x \psi}
$$

If we use (11.1.11), (II.2.20), and the Gauss formula

$$
\widetilde{R}_{a b} \widetilde{n}^{a} \widetilde{n}^{b}-\frac{1}{2} \widetilde{R}=\frac{1}{2}\left[\widetilde{H}^{2}-\widetilde{H}^{\alpha \beta} \widetilde{H}_{\alpha \beta}-\mathcal{R}\right]
$$

where $\mathcal{R}$ is the scalar curvature of the slice $V_{x}$ in the induced metric, then we can eliminate $\widetilde{R}_{a b} \widetilde{n}^{a} \widetilde{n}^{b}$ and $\widetilde{R}$ to obtain

$$
\widetilde{H}=\frac{x \psi}{(n-2)}\left[\frac{x}{2(n-1)} \frac{\partial \widetilde{R}}{\partial x}+\mathcal{R}-\widetilde{H}^{2}+\widetilde{H}_{\alpha \beta} \widetilde{H}^{\alpha \beta}\right] .
$$

From point ( $i$ ) of Proposition II.2.1 (and using $\mathcal{R}(0)=0$ ), we can write that $\widetilde{H}_{\alpha \beta}=$ $\mathcal{O}\left(x^{n-1}\right), \psi=\ell+\mathcal{O}\left(x^{n}\right)$, and $\mathcal{R}=\mathcal{O}\left(x^{n}\right)$, so

$$
\widetilde{H}=\frac{x^{2} \ell}{2(n-1)(n-2)} \frac{\partial \widetilde{R}}{\partial x}+\mathcal{O}\left(x^{n+1}\right)
$$

Finally, Corollary II.2.2 implies that

$$
\frac{\partial \widetilde{R}}{\partial x}=\frac{x^{n-3}}{(n-3) !} \widetilde{R}^{(n-2)}(0)+\mathcal{O}\left(x^{n-2}\right)
$$

Substitution of (II.2.24) into (II.2.23) yields (II.2.19). 


\section{II.3 The Mass}

We now show that $\widetilde{R}^{(n-2)}(0)$ is, up to a factor, the "mass aspect" whose integral over the conformal boundary agrees with the Ashtekar-Magnon conformal definition of mass. ${ }^{5} \mathrm{In}$ Appendix A, we show that it is similarly related to the Hawking-Horowitz mass. Equivalence to various other AdS mass definitions then follows [21]. In view of Corollary II.2.3, this establishes that the sign of the mass aspect governs the mean convexity/concavity of constant $x$ surfaces near infinity.

For any spacetime of dimension $n+1 \geq 4$ with metric $g_{a b}$ and Riemann tensor $P^{a}{ }_{b c d}$, the Weyl tensor $C_{a b c d}$ is defined by

$$
\begin{aligned}
C_{a b c d} & :=P_{a b c d}-\frac{1}{n-1}\left(g_{b c} S_{a d}-g_{b d} S_{a c}+g_{a d} S_{b c}-g_{a c} S_{b d}\right), \\
S_{a b} & :=P_{a b}-\frac{1}{2 n} g_{a b} P .
\end{aligned}
$$

Under the conformal transformation $\widetilde{g}_{a b}=\Omega^{2} g_{a b}, S_{a b}$ obeys

$$
S_{a b}=\widetilde{S}_{a b}+\frac{(n-1)}{\Omega} \widetilde{\nabla}_{a} \widetilde{\nabla}_{b} \Omega-\frac{(n-1)}{2 \Omega^{2}} \widetilde{g}_{a b} \widetilde{g}^{c d} \widetilde{\nabla}_{c} \Omega \widetilde{\nabla}_{d} \Omega
$$

We are interested in the particular case where the metric is the conformally rescaled spacetime metric $\widetilde{g}_{a b}:=x^{2} g_{a b}$, with $g_{a b}$ as in equation (II.1.1), so $\Omega=1 / N=: x$. We maintain our convention of having tildes denote quantities defined with respect to a rescaled metric. We observe that $\widetilde{g}_{a b}$ has a unit timelike Killing field, which we denote $\widetilde{t}^{a}, \widetilde{g}_{a b} \widetilde{t}^{a} \widetilde{t}^{b}=-1$, and therefore $\widetilde{P}_{a b c d} t^{a} t^{c}=0$. We now contract (II.3.1) with $\widetilde{t}^{a} \widetilde{n}^{b} \widetilde{t}^{c} \widetilde{n}^{d}$, where $\widetilde{n}^{a}$ is the unit (in $\widetilde{g}_{a b}$ ) normal to the constant $x$ surfaces (thus $\widetilde{g}_{a b} \widetilde{n}^{a} \widetilde{n}^{b}=+1, \widetilde{g}_{a b} \widetilde{t}^{a} \widetilde{n}^{b}=0$ ), obtaining

$$
\frac{1}{n-1}\left(\widetilde{S}_{a b} \widetilde{n}^{a} \widetilde{n}^{b}-\widetilde{S}_{a b} \widetilde{t}^{a} \widetilde{t}^{b}\right)=\widetilde{C}_{a b c d} \widetilde{t}^{a} \widetilde{n}^{b} \widetilde{t}^{c} \widetilde{n}^{d}=\frac{1}{x^{2}} C_{a b c d} t^{a} n^{b} t^{c} n^{d}=\frac{1}{x} E_{a c} t^{a} t^{c}
$$

where $C_{a b c d}$ and $t^{a}=x \widetilde{t^{a}}$ are, respectively, the Weyl tensor and timelike Killing field of the unrescaled spacetime metric, and $E_{a b}$ is the electric part of $C_{a b c d}$ with respect to $n^{a}=x \widetilde{n}^{a}{ }^{6}$

To evaluate the left-hand side, we use the condition that the spacetime metric $g_{a b}$ is Einstein, which here we can write as $S_{a b}=(\Lambda / n) g_{a b}$. Then (II.3.3) becomes

$$
\begin{aligned}
\widetilde{S}_{a b} & =-\frac{(n-1)}{x} \widetilde{\nabla}_{a} \widetilde{\nabla}_{b} x+\frac{1}{2 n x^{2}} \widetilde{g}_{a b}[n(n-1) \widetilde{W}+2 \Lambda] \\
& =\widetilde{R}_{a b}-\frac{1}{2 n} \widetilde{R} \widetilde{g}_{a b},
\end{aligned}
$$

where in the last step we used equations (II.1.4) and (II.1.7). Then it is easy to evaluate the left-hand side of (II.3.4), yielding

$$
\frac{1}{x} E_{a c} t^{a} t^{c}=\frac{1}{n-1}\left(\widetilde{R}_{a b} \widetilde{n}^{a} \widetilde{n}^{b}-\frac{1}{n} \widetilde{R}\right) .
$$

\footnotetext{
${ }^{5}$ In [11, $\widetilde{b}_{\alpha \beta}^{(n)}$ was related to a new definition of mass.

${ }^{6}$ The Ashtekar and Magnon [4] definition of $E_{a b}$ differs from ours by a factor of $\ell^{2}$. This compensates for the fact that they use a normal vector $n^{a}$ of magnitude $1 / \ell$, whereas we use a unit normal.
} 
But we can use the identity (II.1.11) to rewrite the right-hand side, obtaining

$$
\begin{aligned}
E_{a c} t^{a} t^{c} & =\frac{x^{2}}{2 n(n-1)} \frac{\partial \widetilde{R}}{\partial x}=\frac{x^{2}}{2 n(n-1)}\left[\frac{x^{n-3}}{(n-3) !} \widetilde{R}^{(n-2)}(0)+\mathcal{O}\left(x^{n-2}\right)\right] \\
& =\frac{(n-2)}{2(n !)} x^{n-1} \widetilde{R}^{(n-2)}(0)+\mathcal{O}\left(x^{n}\right)
\end{aligned}
$$

where we used Corollary (II.2.2) and assumed that the metric is $C^{n+1}$. We are now in a position to prove the following:

Proposition II.3.1. If $(\Sigma, h, N)$ obeys equations (II.1.2 II.1.3) and is $C^{n+1}$ conformally compactifiable, then the generalized (in dimension $n$ ) Ashtekar-Magnon mass $M_{A M}$ of $\Sigma$ is given by

$$
M_{A M}=\left.\frac{-\ell}{16 \pi n !} \int_{\partial \widetilde{\Sigma}} \frac{\partial^{n-2} \widetilde{R}}{\partial x^{n-2}}\right|_{0} \sqrt{\widetilde{b}} d S=\frac{-\ell \operatorname{vol}(\partial \widetilde{\Sigma})}{16 \pi n !}\left\langle\left.\frac{\partial^{n-2} \widetilde{R}}{\partial x^{n-2}}\right|_{0}\right\rangle,
$$

where $\operatorname{vol}(\partial \widetilde{\Sigma})=\int_{\partial \widetilde{\Sigma}} \sqrt{\widetilde{b}} d S$ and angle brackets denote the average over $\partial \widetilde{\Sigma}$ with respect to the measure $\sqrt{\tilde{b}} d S$.

Proof. Let $t^{a}$ be a timelike Killing vector field of the spacetime metric. Let $\Sigma, \partial \widetilde{\Sigma}$ be as above. Then we define the Ashtekar-Magnon mass (cf. [4], eq. (11) for the $n=3$ case) by

$$
M_{A M}:=\frac{-\ell}{8 \pi(n-2)} \int_{\partial \widetilde{\Sigma}} E_{a b} t^{a} t^{b} \sqrt{b} d S
$$

where $\sqrt{b} d S=\sqrt{\widetilde{b}} d S / x^{n-1}$ is the measure induced on $\partial \widetilde{\Sigma}$ by the (unrescaled) metric on a constant $x$ surface (so $\sqrt{\widetilde{b}} d S$ is the measure induced by the Fermat metric) and the limit $x \rightarrow 0$ is to be taken. Since we assume the metric is $C^{n}$, we may use (II.3.7), from which we obtain

$$
M_{A M}=\frac{-\ell}{16 \pi n !} \int_{\partial \widetilde{\Sigma}} \widetilde{R}^{(n-2)}(0) \sqrt{\widetilde{b}} d S
$$

in the limit as $x \rightarrow 0$.

Definition II.3.2. In light of Proposition II.3.1, we define the mass aspect of a static, negative mass spacetime with Ricci flat conformal boundary at $x=0$ to be

$$
\mu:=\frac{-\ell}{2(n !)} \widetilde{R}^{(n-2)}(0)
$$

Then notice from Corollary II.2.3 that negative mass aspect implies that surfaces $x=\epsilon=$ const are mean (outward) convex for small enough $\epsilon>0$.

\section{A Geometric Structure Theorem and Negative Mass}

En route to the uniqueness theorem for the soliton, we will obtain a more general structure result for static spacetimes that obey a convexity condition on the extrinsic geometry of constant lapse surfaces near scri. Section III.1 discusses the convexity condition and relates it to negative mass, while III.2 contains the structure theorem and its proof. 


\section{III.1 Convex Surfaces of Constant Lapse}

Consider the level surfaces of the lapse $N$ (equivalently, of $x:=\widetilde{N}:=1 / N$ ). Recall that the second fundamental form $\widetilde{H}_{\alpha \beta}$ of each level surface is defined in Section II using the Fermat "outward" unit normal vector pointing towards scri. The eigenvalues of $\widetilde{H}_{\alpha \beta}$ are called the principal curvatures.

Definition III.1.1. We say that $(\Sigma, h, N)$ satisfies condition $(S)$ provided that the second fundamental form $\widetilde{H}_{\alpha \beta}$ of each level surface $N=c$ is semi-definite (equivalently, provided that the principal curvatures of each level surface $N=c$ are either all non-negative or all nonpositive) whenever $c$ is sufficiently large (i.e., near scri). If $\widetilde{H}_{\alpha \beta}$ is positive semi-definite (equivalently, if the principal curvatures are all non-negative) for each of the level surfaces in this neighbourhood of scri, we say that $(\Sigma, h, N)$ satisfies condition $(C)$, and the level surfaces of $N$ in this neighbourhood are said to be weakly convex.

In the next subsection, we will use Condition (C) to control the behaviour of certain geodesics near scri as follows. Suppose Condition (C) holds, so that the level surfaces $N=c$ are weakly convex, in the sense of the definition, for all $c$ sufficiently large. Let $V=\left\{N=c_{0}\right\}$ be such a level surface; $V$ has a well defined "inside" $\left(N<c_{0}\right)$ and "outside" $\left(N>c_{0}\right)$. Then, as follows from the maximum principle, if $\gamma$ is a geodesic segment with endpoints inside $V$, all of $\gamma$ must be contained inside $V$. Thus, Condition (C) provides "barrier surfaces" for the construction of certain minimizing geodesics, as will be seen in the next subsection.

Condition (S) allows the level surfaces $N=c$ near scri to be either mean convex $\left(H_{\alpha \beta}\right.$ positive semi-definite) or mean concave $\left(H_{\alpha \beta}\right.$ negative semi-definite). All the relevant examples known to us obey condition $(\mathrm{S})$, even when Condition $(\mathrm{C})$ fails. As the following lemma indicates, when Condition (S) holds, the sign of the mass aspect $\mu$ determines, in the case of interest here, whether one gets weakly convex or weakly concave surfaces.

Lemma III.1.2. If $\mu<0$ pointwise on the Ricci flat conformal boundary at $x=0$ of a $C^{n+1}$ conformally compactifiable spacetime and if Condition $(S)$ holds, then Condition (C) holds.

Proof. From Corollary II.2.3 and Definition III.1.1, we have that $\mu<0$ implies $\widetilde{H}(1 / c)>0$ whenever $c>C$ for some $C \in \mathbb{R}$, so the sum of the principal curvatures of each $x=1 / c$ level set is positive. By Condition (S), the principal curvatures are either all nonnegative or all nonpositive, so that sign is nonnegative, implying Condition (C).

\section{III.2 The Structure Theorem}

Theorem III.2.1. Consider an $(n+1)$-dimensional static spacetime as in (1I.1.1) such that $(i)(\Sigma, h, N)$ is smoothly $\left(C^{\infty}\right)$ conformally compactifiable, (ii) the static vacuum field equations hold, and (iii) condition $(C)$ holds. Then the Riemannian universal cover $\left(\widetilde{\Sigma}^{*}, \widetilde{h}^{*}\right)$ of the conformally related spacetime $(\widetilde{\Sigma}, \widetilde{h})$ splits isometrically as

$$
\widetilde{\Sigma}^{*}=\mathbb{R}^{k} \times \widetilde{W}, \quad \widetilde{h}^{*}=h_{E} \oplus \tilde{\sigma}
$$

where $\left(\mathbb{R}^{k}, h_{E}\right)$ is standard $k$-dimensional Euclidean space, with $0 \leq k \leq n$, and $(\widetilde{W}, \tilde{\sigma})$ is a compact Riemannian manifold with non-empty boundary. The Riemannian universal 
cover $\left(\Sigma^{*}, h^{*}\right)$ of $(\Sigma, h)$ splits isometrically as a warped product of the form

$$
\Sigma^{*}=\mathbb{R}^{k} \times W, \quad h^{*}=\left(N^{* 2} h_{E}\right) \oplus \sigma,
$$

where $N^{*}=N \circ \pi\left(\pi=\right.$ covering map) is constant on $\mathbb{R}^{k}$, and $(W, \sigma)$ is a simply connected Riemannian manifold such that $(W, \sigma, N)$ is smoothly conformally compactifiable.

Remark III.2.2. Notice that we specify no boundary data in this theorem, instead imposing only the convexity condition (C). In particular, this theorem does not require Ricci flatness of the conformal boundary. However, if the conformal boundary is Ricci flat and if the mass is negative, we may relax Condition (C) to Condition (S), obtaining in this case a structure theorem for negative mass static spacetimes.

Remark III.2.3. Theorem III.2.1 is similar in spirit to the Cheeger-Gromoll splitting theorem [7], or more precisely, to Theorem 3.16 in [34, a structure theorem for compact (without boundary) Riemannian manifolds of nonnegative Ricci curvature, which is a direct consequence of the Cheeger-Gromoll splitting theorem. Theorem III.2.1 implies a strong structure result for the fundamental group $\pi_{1}(\widetilde{\Sigma})$ of $\widetilde{\Sigma}$. For example, if $k=0$ then the universal cover is compact, and $\pi_{1}(\widetilde{\Sigma})$ is finite. More generally, Theorem III.2.1 implies that $\pi_{1}(\tilde{\Sigma})$ is "almost abelian", i.e., that there exists a finite normal subgroup $F$ of $\pi_{1}(\tilde{\Sigma})$ such that $\pi_{1}(\tilde{\Sigma}) / F$ contains a subgroup of finite index isomorphic to $\mathbb{Z}^{k}$, cf. [7].

Proof. The proof of this theorem consists of three parts. We first show that a null line exists in the universal covering spacetime whenever $\Sigma^{*}$ is noncompact. The null splitting theorem then tells us that this null line lies in a smooth, closed, achronal, totally geodesic null hypersurface $\mathcal{H}$. Staticity then implies that $\mathcal{W}_{t}=\Sigma_{t}^{*} \cap \mathcal{H}$ is totally geodesic, where $\Sigma_{t}^{*}$ is a constant time slice. The $t=0$ slice $\Sigma_{0}^{*}$ can thus be foliated by the projections of the $\mathcal{W}_{t}$ into it. Using (1.1.2 ஹ.1.3), we show that we can then isometrically split off an $\mathbb{R}$ factor, and continuing iteratively yields the result.

(i) Construction of a null line:

A line in a Riemannian manifold is a complete geodesic, each segment of which is minimal (length minimizing). We describe here how to construct a line in $\left(\widetilde{\Sigma}^{*}, \widetilde{h}^{*}\right)$, provided $\widetilde{\Sigma}^{*}$ is noncompact. We then make use of a fundamental feature of the Fermat metric: Length minimizing Fermat geodesics lift in an essentially unique way to achronal null geodesics in the physical spacetime, see Appendix B.

Let $\left(\widetilde{\Sigma}^{*}, \widetilde{h}^{*}\right)$ be the Riemannian universal cover of $(\widetilde{\Sigma}, \widetilde{h})$. If $\widetilde{\Sigma}^{*}$ is compact, then $k=0$ in the above splitting and we are done, so assume otherwise. Let $p \in \operatorname{int}\left(\widetilde{\Sigma}^{*}\right)$ and let $\left\{q_{i}\right\}$ be a sequence of points bounded away from $\partial \widetilde{\Sigma}^{*}$, such that the distances from $p$ to successive $q_{i}$ tend to infinity. For each $i$, let $\gamma_{i}$ be a minimal geodesic from $p$ to $q_{i}$. The convexity condition (C) implies that the $\gamma_{i}$ 's are uniformly bounded away from $\partial \widetilde{\Sigma}^{*}$. Fix a fundamental domain $D \subseteq \Sigma^{*}$. For each midpoint $r_{i}$ of $\gamma_{i}$, there is a covering space transformation $\mathfrak{g}_{i} \in \pi_{1}(\widetilde{\Sigma})$, possibly the identity, mapping $r_{i}$ into $D$. Because the $\mathfrak{g}_{i}$ are discrete isometries, the images $\hat{\gamma}_{i}=\gamma_{i} \circ \mathfrak{g}_{i}$ form a sequence of minimal geodesics that all meet $D$ and remain uniformly bounded away from $\partial \widetilde{\Sigma}^{*}$. Since $\widetilde{\Sigma}$ is compact then so is $D$, and so there will be a convergent subsequence of the $\hat{\gamma}_{i}$ whose limit is a complete, minimal geodesic (thus a line) $\gamma$ of the Fermat metric $\widetilde{h}^{*}$, meeting $D$, which is bounded away from $\partial \widetilde{\Sigma}^{*}$. Finally, fix a point on $\gamma$. By Lemma B.1 in Appendix B, through that point there is 
a unique, future directed null line $\eta$ in $\left(\Sigma^{*}, h^{*}, N^{*}\right)$ produced by lifting $\gamma$ along the timelike Killing field.

(ii) Splitting off one $\mathbb{R}$ factor:

Let $\lambda \rightarrow \mu(\lambda), \lambda \in I$, be an inextendible null geodesic in the physical spacetime $(\mathcal{M}, g)$. Using the constant of motion lemma with respect to the Killing field $\partial / \partial t$ and the fact that $\mu$ is null, one obtains along $\mu$ that $d \lambda=N d s$ (up to a factor constant along $\mu$ ) and $s$ is $h$-arclength along the projection of $\mu$ into $\Sigma$. Note that since $(\Sigma, h, N)$ is conformally compactifiable, $(\Sigma, h)$ is necessarily geodesically complete and $N$ is bounded positively away from zero. From the geodesic completeness of $(\Sigma, h)$ and the inextendibility of $\mu$, it follows that $s \rightarrow \pm \infty$ as $\lambda$ ranges over $I$. Since $N$ is bounded away from zero, the equation $d \lambda=N d s$ then implies that $\lambda$ ranges over all real numbers, i.e., $I=\mathbb{R}$. Thus, $(\mathcal{M}, g)$ is null geodesically complete, and this completeness lifts to the universal covering spacetime.

Because the null geodesically complete covering spacetime $\left(\Sigma^{*}, h^{*}, N^{*}\right)$ satisfies the null energy condition and admits the null line $\eta$, the null splitting theorem I.1 implies that $\eta$ is contained in a smooth, connected, achronal, edgeless totally geodesic null surface $\mathcal{H}$. By the constant of motion lemma, the equation $d t / d \lambda=E /\left(N^{*}\right)^{2}$ holds along $\lambda \mapsto \eta(\lambda)$, where $\lambda$ is an affine parameter, $E$ is constant along $\eta$, and, because $\gamma$ is bounded away from $\partial \widetilde{\Sigma}^{*}, N^{*}$ is bounded along $\eta$. Since $\eta$ is complete it follows that $t$ ranges over all real numbers. Thus $\eta$ meets each constant $t$ hypersurface $\Sigma_{t}^{*}=\{t\} \times \Sigma^{*}$. Since each $\Sigma_{t}^{*}$ is totally geodesic, the codimension 2 spacelike intersections $\mathcal{W}_{t}=\Sigma_{t}^{*} \cap \mathcal{H}$ are also totally geodesic.

Let $\mu$ be any other null generator of $\mathcal{H}$ passing through $\mathcal{W}_{0}$. Since $\mathcal{H}$ is totally geodesic, its null generators have zero expansion and shear. It follows that the spatial separation of $\mu$ and $\eta$ remains constant along these generators. Hence, the projection of $\mu$ into $\Sigma^{*}$ will also be bounded away from $\partial \widetilde{\Sigma}^{*}$, and by the same argument as above, $t$ will range over all reals along $\mu$. Thus, the null generators of $\mathcal{H}$ meeting $\mathcal{W}_{0}$ can be parametrized by time $t \in \mathbb{R}$ so as to define a flow $\mathcal{F}: \mathbb{R} \times \mathcal{W}_{0} \rightarrow \mathcal{H}$ along $\mathcal{H}$. Using that $\mathcal{H}$ is connected and closed, it can be shown that $\mathcal{F}$ is onto. Hence, fixing any $t \in \mathbb{R}$, the flow induces a diffeomorphism $\mathcal{W}_{0} \rightarrow \mathcal{W}_{t}$.

Let $\mathcal{P}$ denote projection into $\Sigma_{0}^{*}$ along integral curves of $\partial / \partial t$ and let $W_{t}:=\mathcal{P}\left(\mathcal{W}_{t}\right)$ (note that $W_{0} \equiv \mathcal{W}_{0}$ ). The composition of $\mathcal{F}$ with $\mathcal{P}$ defines a flow $F:=\mathcal{P} \circ \mathcal{F}: \mathbb{R} \times W_{0} \rightarrow$ $\Sigma_{0}^{*}$ on the $t=0$ hypersurface. The $t$ parametrization on $\mathcal{F}$ gives a parametrization for $F$ by arclength in the Fermat metric $\widetilde{h}^{*}:=h^{*} /\left(N^{*}\right)^{2}=(\mathcal{P} g) /\left(N^{*}\right)^{2}$. The equipotentials are $\mathcal{P}\left(\mathcal{W}_{t}\right) \equiv W_{t}$ and are all diffeomorphic copies of $W_{0}$. The flow lines $t \mapsto F(t, q)$, $q \in W_{0}$, being projections of the null generators of $\mathcal{H}$, are Fermat geodesics orthogonal to the $W_{t} \mathrm{~s}$. We prefer to relabel the parameter along the flow $F$ as $u$ from here onward, so we write the equipotentials as $W_{u}$ and the flow field orthogonal to them as $\partial / \partial u$. Since $\left.\mathcal{P}\right|_{\mathcal{H}}$ is both an open and closed mapping, it follows that $\mathcal{P}(\mathcal{H})=\Sigma_{0}^{*}$. Thus, $F$ maps $\mathbb{R} \times W_{0}$ diffeomorphically onto $\Sigma_{0}^{*}$, i.e., $\Sigma_{0}^{*} \approx \mathbb{R} \times W_{0}$. Finally, we pull this back along the embedding $i: \Sigma^{*} \rightarrow \Sigma_{0}^{*}$ to obtain $\Sigma^{*} \approx \mathbb{R} \times W$ where $W_{0}=i(W)$. Thus, we have extracted the first topological factor of $\mathbb{R}$. We now turn our attention to the geometrical splitting.

In coordinates adapted to the flow, which in fact are just Gaussian normal coordinates 
about $W_{0}$, the Fermat metric takes the form

$$
\widetilde{h}^{*}=d u^{2}+\tilde{\sigma}_{i j} d y^{i} d y^{j}
$$

where $u \in \mathbb{R}, \vec{y}$ represents coordinates on $W_{0}$, and $\tilde{\sigma}_{i j}(u, \vec{y})$ is the induced metric on $W_{0}$. The physical metric $h^{*}$ is then

$$
h^{*}=N^{* 2}(u, \vec{y}) d u^{2}+\sigma_{i j}(\vec{y}) d y^{i} d y^{j},
$$

where, since each $W_{u}$ is totally geodesic in the physical metric, the induced metric $\sigma_{i j}(\vec{y})=$ $N^{* 2} \tilde{\sigma}_{i j}$ on $W_{0}$ is $u$-independent.

We now show that the lapse $N^{*}$ is independent of $u$. Let $N^{a}=\frac{1}{N^{*}}\left(\frac{\partial}{\partial u}\right)^{a}$ be the unit normal to the surfaces $W_{u}$. Since the $W_{u}$ are totally geodesic, the Codazzi relation yields $R_{a b}^{*} N^{a} h_{c}^{* b}=0$, so that $R_{a b}^{*} N^{a} Y^{b}=0$ for any $Y^{a}$ tangent to $W_{u}$. Using this in eq. (II.1.2), we get

$$
N^{a} Y^{b} \nabla_{a} \nabla_{b} N^{*}=0 \quad, \quad \forall Y^{a} \in T W_{u} .
$$

But the surfaces $W_{u}$ are totally geodesic, so the Hessian in (III.2.5) becomes the double directional derivative $\nabla_{N}\left(\nabla_{Y} N^{*}\right)$. Since this vanishes for all $Y^{a} \in T W_{u}$, the general solution is $N^{*}=\alpha(u) \beta(\vec{y})$. Contracting equation (II.1.2) with $N^{a} N^{b}$ yields,

$$
\begin{aligned}
R_{a b}^{*} N^{a} N^{b} & =\frac{1}{\alpha^{2} \beta^{2}} R_{u u}^{*} \\
& =\frac{1}{\alpha^{2} \beta^{2}}\left(\frac{1}{\alpha} \frac{d^{2} \alpha}{d u^{2}}-\frac{1}{\alpha^{2}}\left(\frac{d \alpha}{d u}\right)^{2}+\alpha^{2} \sigma^{i j} \frac{\partial \beta}{\partial y^{i}} \frac{\partial \beta}{\partial y^{j}}+\frac{2 \Lambda}{(n-1)} \alpha^{2} \beta^{2}\right) .
\end{aligned}
$$

Since $N=\alpha(u) \beta(\vec{y})$, we can make the coordinate transformation $v=\int \alpha d u$, and since $\alpha>0$ is uniformly bounded above and away from zero below and $u$ takes values throughout $\mathbb{R}$, so does $v$. Notice that $\left(\frac{\partial}{\partial v}\right)^{a}$ is a Killing vector in $h^{*}$ (but not necessarily in the full spacetime). In the new coordinates $(v, \vec{y})$, we have $R_{u u}^{*}=\alpha^{2} R_{v v}^{*}$, where $R_{v v}^{*}$ is independent of $v$. Eq. (III.2.6) is thus separable in $v$ and $\vec{y}$, and takes the form

$$
R_{v v}^{*}-\sigma^{i j} \frac{\partial \beta}{\partial y^{i}} \frac{\partial \beta}{\partial y^{j}}-\frac{2 \Lambda}{(n-1)} \beta^{2}=\frac{1}{\alpha^{3}} \frac{d^{2} \alpha}{d u^{2}}-\frac{1}{\alpha^{4}}\left(\frac{d \alpha}{d u}\right)^{2}=\frac{1}{\alpha(v)} \frac{d^{2} \alpha}{d v^{2}}
$$

yielding in particular

$$
\frac{1}{\alpha(v)} \frac{d^{2} \alpha}{d v^{2}}=c
$$

where $c$ is the separation constant. Obviously the only solution suitably bounded on all of $\mathbb{R}$ is $\alpha=$ const., which occurs only for $c=0$. This in turn implies that the lapse $N^{*}$ is independent of $u$, which implies that the $W_{u}$ are totally geodesic in the Fermat metric.

The spacetime $\left(\Sigma^{*}, h^{*}, N^{*}\right)$ therefore splits as $\Sigma^{*}=\mathbb{R} \times W$, and $h^{*}=N^{* 2} d u^{2} \oplus \sigma$, where $N^{*}$ and $\sigma$ are $u$-independent. $\left(\widetilde{\Sigma}^{*}, \widetilde{h}^{*}, \widetilde{N}^{*}\right)$ then splits as a product $\widetilde{\Sigma}^{*}=\mathbb{R} \times \widetilde{W}$, $\widetilde{h}^{*}=d u^{2} \oplus \tilde{\sigma}$ (the splitting clearly extending to the boundary). 
(iii) Iteration:

If $\widetilde{W}$ is compact then we are done and $k=1$. If not, we proceed inductively. Assume that we have split off $p$ factors of $\mathbb{R}$, so that

$$
\widetilde{\Sigma}^{*}=\mathbb{R}^{p} \times \widetilde{W} \quad, \quad \widetilde{h}^{*}=\left(\sum_{j=1}^{p} d u_{j}^{2}\right) \oplus \tilde{\sigma}_{(n-p)} \quad, \quad \frac{\partial N^{*}}{\partial u_{j}}=0
$$

and $\widetilde{W}$ is noncompact. We then proceed to split off another factor of $\mathbb{R}$ as follows.

Let $\widetilde{W}_{(n-p)}=\bigcap_{j=1}^{p}\left\{u_{j}=0\right\} \cap \widetilde{\Sigma}_{0}^{*}$. We first show that since $\widetilde{W}_{(n-p)}$ is noncompact, not all the lines in $\widetilde{\Sigma}_{0}^{*}$ can lie in $\mathbb{R}^{p}$. Let us assume otherwise, i.e., that all lines in $\widetilde{\Sigma}_{0}^{*}$ have the form $\gamma(s)=\left(\gamma_{1}(s), x\right)$, where $\gamma_{1}(s)$ is a line in $\mathbb{R}^{p}$ and $x \in \widetilde{W}_{(n-p)}$. Under the covering space isometry, $\mathfrak{g} \in \pi_{1}(\widetilde{\Sigma})$, lines get mapped to lines. Now, each vector in the tangent space of $\mathbb{R}^{p}$ is tangent to a line in $\mathbb{R}^{p}$. By assumption, all the lines lie in the $\mathbb{R}^{p}$ factor, so that the endomorphism $\varphi$ on $T \widetilde{\Sigma}_{0}^{*}$ induced by $\mathfrak{g}$ preserves the tangent space $T \mathbb{R}^{p} \subset T \widetilde{\Sigma}_{0}^{*}$. Since $T \widetilde{W}_{(n-p)}$ is orthogonal to $T \mathbb{R}^{p}$ and $\varphi$ is linear, $\varphi$ preserves $T \widetilde{W}_{(n-p)}$ as well. Since $\widetilde{W}_{(n-p)}$ is noncompact, we may repeat the construction, as in part $(i)$, of a sequence of minimal geodesic segments $\gamma_{i}$ in $\widetilde{W}_{(n-p)}$, whose lengths diverge to infinity, and which are uniformly bounded away from $\partial \widetilde{W}_{(n-p)}$. Again, we use the covering transformations $\mathfrak{g}_{i}$ to map the midpoints $r_{i}$ of $\gamma_{i}$, to the fundamental domain $D$ of $\widetilde{\Sigma}_{0}^{*}$. As before, the minimal geodesics $\gamma_{i}^{\prime}=\gamma_{i} \circ \mathfrak{g}_{i}$ meet the compact set $D$, and, by passing to a subsequence if necessary, converge to a line $\gamma$. By assumption, $\gamma$ lies in the factor $\mathbb{R}^{p}$. Since $\mathfrak{g}_{i}$ maps the velocity vectors of $\gamma_{i}$ to the velocity vectors of $\gamma_{i}^{\prime}$, it follows that for large $i, \mathfrak{g}_{i}$ maps vectors tangent to $\widetilde{W}_{(n-p)}$ to vectors nearly perpendicular to $\widetilde{W}_{(n-p)}$. This is a contradiction, so we conclude that not all lines lie in $\mathbb{R}^{p}$.

Since the $u_{j}$-directions are flat in the Fermat metric any line $\gamma:(-\infty, \infty) \rightarrow \widetilde{\Sigma}_{0}^{*}=$ $\mathbb{R}^{p} \times \widetilde{W}_{(n-p)}$ itself splits as $\gamma(s)=\left(\gamma_{1}(s), \gamma_{2}(s)\right)$, where $\gamma_{1}$ is a line in $\mathbb{R}^{p}$ and $\gamma_{2}$ is a line in $\widetilde{W}_{(n-p)}$-or possibly one of $\gamma_{1}, \gamma_{2}$ is constant, but since not all lines lie in the $\mathbb{R}^{p}$ factor, $\left(\widetilde{W}_{(n-p)}, \tilde{\sigma}_{(n-p)}\right)$ itself must contain at least one line. Call it $\widetilde{\zeta}$. Since the hypersurfaces $u_{j}=0$ are all timelike, $\left(W_{(n-p)}, \sigma_{(n-p)}, N^{*}\right)$ is a static spacetime $\mathcal{S}$ in its own right (even if it may not satisfy equations (II.1.2 II.1.3), preventing us from applying the null splitting theorem directly). Thus, $\widetilde{\zeta}$ can be lifted to a null line $\zeta$ in $\mathcal{S}$. Because the covering spacetime $\left(\Sigma^{*}, h^{*}, N^{*}\right)$ is the metric product $\mathbb{R}^{p} \times \mathcal{S}, \zeta$ is also a null line in $\left(\Sigma^{*}, h^{*}, N^{*}\right)$. The null splitting theorem can now be applied to show that $\zeta$ lies in a smooth, edgeless, achronal, totally geodesic null hypersurface $\mathcal{H}_{p+1}$.

We now apply the construction of part $(i i)$ to $\mathcal{H}_{p+1}$ to produce a foliation of $\widetilde{W}_{(n-p)}$. As in part $(i i)$, there is a null geodesic flow on $\mathcal{H}_{p+1}$ that projects to a flow on $\widetilde{\Sigma}_{0}^{*}$. We must show that this flow is tangent to $\widetilde{W}_{(n-p)}$. Equivalently, the flow on $\mathcal{H}_{p+1}$ must be tangent to $\mathcal{S}$. But this follows because $\zeta \subset \mathcal{S}$, so $\dot{\zeta}^{a} \in T \mathcal{S}$, and the flow vector field on $\mathcal{H}_{p+1}$ to which $\dot{\zeta}^{a}$ belongs is parallel (cf. Remark I.2(b)).

We thus obtain the splitting $\left(\widetilde{W}_{(n-p)}, \tilde{\sigma}_{(n-p)}\right)=\left(\mathbb{R} \times \widetilde{W}_{(n-p-1)}, d u_{p+1}^{2} \oplus \tilde{\sigma}_{(n-p-1)}\right)$, with $\tilde{\sigma}$ independent of $u_{p+1}$, or $\left(W_{(n-p)}, \sigma_{(n-p)}\right)=\left(\mathbb{R} \times W_{(n-p-1)}, N^{* 2} d u_{p+1}^{2} \oplus \sigma_{(n-p-1)}\right)$. 
Repeating the arguments of part (ii), we obtain that $N^{*}$ is $u_{p+1}$-independent, and thus so is $\sigma$. Hence we write

$$
h^{*}=\left(N^{* 2} \sum_{j=1}^{p+1} d u_{j}^{2}\right) \oplus \sigma \quad, \quad \frac{\partial N^{*}}{\partial u_{j}}=0 \quad, \quad j=1, \ldots, p+1,
$$

where $\sigma$ is independent of the $u_{j}$. Since this procedure is valid for any $p=1, \ldots, n$, one can continue splitting off $\mathbb{R}$ factors until what remains is compact.

\section{The Uniqueness Theorem}

Theorem IV.1. Consider a static spacetime as in (II.1.1) such that $(\Sigma, h, N)$ (1) is smoothly conformally compactifiable, (2) satisfies the static vacuum field equations, and (3) satisfies condition ( $S$ ). In addition, assume the following conditions to hold:

(a) The boundary geometry of $(\widetilde{\Sigma}, \widetilde{h})$ is the same as that of (I.1) , i.e., $\partial \widetilde{\Sigma}=T^{n-2} \times S^{1}$, $\left.\widetilde{h}\right|_{\partial \tilde{\Sigma}}=\sum_{i=1}^{n-2}\left(d \theta^{i}\right)^{2}+\frac{1}{\ell^{2}} d \phi^{2}$.

(b) The mass aspect $\mu$ of $(\Sigma, h, N)$ is pointwise negative.

(c) Given the inclusion map $i: \partial \widetilde{\Sigma} \rightarrow \widetilde{\Sigma}$, the kernel of the induced homomorphism of fundamental groups, $i_{*}: \pi_{1}(\partial \widetilde{\Sigma}) \rightarrow \pi_{1}(\widetilde{\Sigma})$, is generated by the $S^{1}$ factor.

Then the spacetime (II.1.1) determined by $(\Sigma, h, N)$ is isometric to the Horowitz-Myers soliton (1.1).

Assumptions (a) and (b) are natural boundary conditions for the uniqueness problem. (Recall from Section II that the mass aspect is related to the free boundary data.) As shown in 9], in $3+1$ dimensions, (b) automatically holds, provided $\mu$ is constant. Assumption (c) pertains to a distinctive topological feature of the AdS soliton. It asserts that the generator of the $S^{1}$ factor in $\left(\partial \widetilde{\Sigma},\left.\widetilde{h}\right|_{\partial \tilde{\Sigma}}\right)$ is contractible in $\widetilde{\Sigma}$, and moreover, that any loop in $\partial \widetilde{\Sigma}$ contractible in $\widetilde{\Sigma}$ is a multiple of the generator. As shown in the proof, assumptions (a) and (c) together imply that $\pi_{1}(\widetilde{\Sigma}) \approx \mathbb{Z}^{n-2}$. Were we to adopt the latter condition en lieu of assumption (c), without assuming the geometrical $S^{1}$ factor is contractible in the bulk, then one could still conclude via the following proof that $(\Sigma, h, N)$ is locally isometric to the AdS soliton (the universal covers will be isometric). For example, this situation can arise when some loop not in the $\partial \widetilde{\Sigma}$ homotopy class of the geometrical $S^{1}$ factor (i.e., some loop not homotopic to an integral curve of $\partial / \partial \phi$ ) is contractible in $\widetilde{\Sigma}$. For further discussion of this issue see Remark IV.3. We note that in $3+1$ dimensions, the condition $\pi_{1}(\widetilde{\Sigma}) \approx \mathbb{Z}^{n-2}$ holds automatically, cf. [15].

Proof. There are two main parts to this proof. First, using the splitting theorem and the topological assumption (c), we show that the number of $\mathbb{R}$ factors in the splitting is precisely $n-2$. This means that the submanifold $W$ is 2 -dimensional. Using the topological censorship theorem [15, we then show that $\widetilde{\Sigma} \simeq T^{n-2} \times W$. The only undetermined functions are then the 2-dimensional metric $\tilde{\sigma}_{A B}(\vec{y})$ on $W$ and the lapse $\widetilde{N}(\vec{y})$, which, with the aid of the field equations, can be solved for explicitly. 


\section{(i) The product structure of $\widetilde{\Sigma}$ :}

As discussed in (III.1), the assumption of negative mass along with condition (S) implies condition (C). Thus, we can invoke the splitting theorem which tells us that there are $k$ noncompact directions in the universal covering space $\widetilde{\Sigma}^{*}$, so that $\widetilde{\Sigma}^{*}=\mathbb{R}^{k} \times W$, where $W$ is compact. By results on topological censorship [15], the homomorphism $i_{*}: \pi_{1}(\partial \widetilde{\Sigma}) \rightarrow$ $\pi_{1}(\widetilde{\Sigma})$ is onto. But $\partial \widetilde{\Sigma}=A \times B$, where $A$ is the $(n-2)$ torus and $B$ is the circle of assumption (a), whence $\pi_{1}(\partial \widetilde{\Sigma}) \approx \pi_{1}(A) \times \pi_{1}(B)$. Since, by assumption (c), ker $i_{*}=\pi_{1}(B)$, it follows that $\left.i_{*}\right|_{\pi_{1}(A)}: \pi_{1}(A) \rightarrow \pi_{1}(\widetilde{\Sigma})$ is an isomorphism, and hence $\pi_{1}(\widetilde{\Sigma}) \approx \mathbb{Z}^{n-2}$. But by Remark III.2.3, $\pi_{1}(\widetilde{\Sigma})$ must contain a subgroup of finite index isomorphic to $\mathbb{Z}^{k}$, from which it follows that $k=n-2$.

Thus, the universal cover $\widetilde{\Sigma}^{*}$ splits isometrically as $\mathbb{R}^{n-2} \times W$. $W$ is therefore a 2dimensional, compact, simply connected manifold with boundary and is thus diffeomorphic to a disk. The covering isometries of $\widetilde{\Sigma}^{*}$ split as $\mathfrak{g}=\left(\mathfrak{g}^{\prime}, \mathfrak{g}^{\prime \prime}\right)$, where $\mathfrak{g}^{\prime}$ and $\mathfrak{g}^{\prime \prime}$ are isometries of $\mathbb{R}^{n-2}$ and $W$, respectively. Let $A^{*} \simeq \mathbb{R}^{n-2}$ denote the universal cover of $A$. Then $A^{*} \times B$ is the covering of $A \times B$ associated with the subgroup $\pi_{1}(A) \subset \pi_{1}(A \times B)$. Since $\left.i_{*}\right|_{\pi_{1}(A)}: \pi_{1}(A) \rightarrow \pi_{1}(\widetilde{\Sigma})$ is an isomorphism, the group of covering isometries of $\widetilde{\Sigma}^{*}$ is naturally isomorphic to $\pi_{1}(A)$. By this isomorphism, the covering isometries of $\widetilde{\Sigma}^{*}$ restrict on $\partial \widetilde{\Sigma}^{*} \simeq \mathbb{R}^{n-2} \times S^{1}$ to the covering isometries of $A^{*} \times B$. Since the latter fix the circle $B$, the former fix the disk $W$, i.e., the covering isometries of $\widetilde{\Sigma}^{*}$ act only on the $\mathbb{R}^{n-2}$ factor. It follows that

$$
\widetilde{\Sigma} \simeq\left(\mathbb{R}^{n-2} \times W\right) / \pi_{1}(A) \simeq\left(\mathbb{R}^{n-2} / \pi_{1}(A)\right) \times W \simeq A \times W \simeq T^{n-2} \times D^{2},
$$

where $D^{2}$ is a closed 2-disc and $T^{n-2}$ is the $(n-2)$-torus of assumption (a).

(ii) Solving for the lapse and metric on the disc:

By the splitting obtained in part (i), the Fermat metric takes the form

$$
d \tilde{s}^{2}=\sum_{i=1}^{n-2}\left(d u^{i}\right)^{2}+\tilde{\sigma}_{i j}(\vec{y}) d y^{i} d y^{j}, \quad \frac{\partial N}{\partial u^{i}}=0,
$$

where $\vec{y}=\left(y^{1}, y^{2}\right)$ and $\tilde{\sigma}_{i j}$ are the coordinates and metric on the disk $W \simeq D^{2}$, respectively. Since there are $n-2$ flat directions, the only contribution to the curvature comes from $K^{m}{ }_{i j k}$, the curvature tensor associated with $\tilde{\sigma}_{i j}$. As $\tilde{\sigma}_{i j}$ is 2-dimensional, the curvature tensor can be expressed as, $K^{m}{ }_{i j k}=\frac{1}{2} K\left(\tilde{\sigma}_{i k} \tilde{\sigma}_{j}{ }^{m}-\tilde{\sigma}_{i}{ }^{m} \tilde{\sigma}_{j k}\right)$, where $K$ is the scalar curvature. The field equations (II.1.4) thus simplify to

$$
\mathbb{D}_{i} \mathbb{D}_{j} \tilde{N}=-\frac{\tilde{N} K}{2(n-1)} \tilde{\sigma}_{i j}
$$

where $\mathbb{D}$ is the covariant derivative compatible with $\tilde{\sigma}_{i j}$. Differentiating, antisymmetrizing, and contracting with $\tilde{\sigma}^{k j}$, we get

$$
\tilde{\sigma}^{k j} \mathbb{D}_{[k} \mathbb{D}_{i]} \mathbb{D}_{j} \widetilde{N}=-\frac{\tilde{\sigma}^{k j}}{2(n-1)} \tilde{\sigma}_{j[i} \mathbb{D}_{k]}(\widetilde{N} K)
$$

which yields the integrability condition $\mathbb{D}_{i}(\tilde{N} K)=(n-1) K \mathbb{D}_{i}(\tilde{N})$. Thus, $K=C \tilde{N}^{n-2}$, where $C$ is an integration constant. 
Let us now consider a neighbourhood $\mathcal{U}$ of the boundary $\partial W \simeq S^{1}$. In this neighbourhood, we can write $\tilde{\sigma}_{i j}$ in Gaussian normal coordinates $(\rho, \phi)$ with respect to the boundary at $\rho=0$ as

$$
d \tilde{\sigma}^{2}=d \rho^{2}+G^{2}(\rho, \phi) d \phi^{2}
$$

Using the condition $K=C \widetilde{N}^{n-2}$, equations (IV.3) become,

$$
\begin{aligned}
\frac{\partial^{2} \tilde{N}}{\partial \rho^{2}} & =-\frac{C}{2(n-1)} \widetilde{N}^{n-1}, \\
\frac{\partial^{2} \widetilde{N}}{\partial \rho \partial \phi} & =\frac{1}{G} \frac{\partial G}{\partial \rho} \frac{\partial \widetilde{N}}{\partial \phi}, \\
\frac{\partial^{2} \widetilde{N}}{\partial \phi^{2}} & =-G \frac{\partial G}{\partial \rho} \frac{\partial \widetilde{N}}{\partial \rho}+\frac{1}{G} \frac{\partial G}{\partial \phi} \frac{\partial \widetilde{N}}{\partial \phi}-\frac{C \widetilde{N}^{n-1} G^{2}}{2(n-1)}
\end{aligned}
$$

The boundary conditions for this system of equations at $\rho=0$ are

$$
G(0, \phi)=\frac{1}{\ell},\left.\quad \frac{\partial \tilde{N}}{\partial \phi}\right|_{\rho=0}=0 \quad,\left.\quad \frac{\partial \tilde{N}}{\partial \rho}\right|_{\rho=0}=\frac{1}{\ell} .
$$

The first of these conditions arises by comparison of (IV.5) and assumption (a) of the Theorem, the second arises since we assume $\widetilde{N}=0$ pointwise on the boundary, and the last is obtained by combining (II.1.6), (II.1.7), (II.2.14), (IV.5), and the second boundary condition. Now, (IV.7) simplifies to

$$
\partial_{\rho}\left(\frac{1}{G} \partial_{\phi} \tilde{N}\right)=0 \Rightarrow \frac{1}{G} \partial_{\phi} \widetilde{N}=\xi(\phi) .
$$

(IV.9) implies that $\xi(\phi)=0$, or $\partial_{\phi} \tilde{N}=0$ everywhere. Thus, (IV.6) reduces to an ordinary differential equation,

$$
\frac{d^{2} \widetilde{N}}{d \rho^{2}}=-\frac{C}{2(n-1)} \widetilde{N}^{n-1}
$$

which integrates to give

$$
\frac{d \widetilde{N}}{d \rho}=\frac{1}{\ell}\left(1-\frac{C \ell^{2} \widetilde{N}^{n}}{n(n-1)}\right)^{1 / 2}
$$

given the boundary conditions (IV.9). Next, combining (IV.6) and (IV.8) and using $\partial_{\phi} \tilde{N}=$ 0 , we get

$$
\partial_{\rho}\left(\frac{1}{G} \partial_{\rho} \tilde{N}\right)=0 \Rightarrow \frac{1}{G} \partial_{\rho} \widetilde{N}=\zeta(\phi)
$$

which along with (IV.9) implies that $\zeta(\phi)=1$, or $\partial_{\phi} G=0$ identically, so that $G(\rho)=\frac{d \widetilde{N}}{d \rho}$.

Using (IV.12) we can rewrite the metric (IV.5) as

$$
d \tilde{\sigma}^{2}=\frac{\ell^{2}}{r^{4}\left(1-\frac{C \ell^{2}}{n(n-1) r^{n}}\right)} d r^{2}+\frac{1}{\ell^{2}}\left(1-\frac{C \ell^{2}}{n(n-1) r^{n}}\right) d \phi^{2} \quad, \quad \widetilde{N}=\frac{1}{r}=\Omega .
$$


Inserting into (IV.2) and comparing with (I.1), we see that the particular identification $\frac{C \ell^{2}}{n(n-1)}=r_{0}^{n}$ yields precisely the soliton metric in the neighbourhood $\mathcal{U}$ of the boundary.

Finally, we note that the assumption of negative mass implies that $r_{0}>0$ and therefore $C>0$. Our coordinates, valid on a neighbourhood of scri, thus extend to all $r>r_{+}^{n}:=$ $\frac{C \ell^{2}}{n(n-1)}$, and the metric is unique on this domain. Now, by the boundary conditions, $\phi$ is periodic with period $\frac{4 \pi \ell^{2}}{n r_{0}}$, and so the further extension to $r=r_{+}$is a cone point singularity, except that it is a smooth point iff we choose $C$ such that $C=C_{0}:=\frac{n(n-1) r_{0}^{n}}{\ell^{2}}$ (as can be seen by the standard technique of expanding $d \tilde{\sigma}^{2}$ in polar coordinates about $r=r_{+}$). Thus, there is a unique nonsingular extension of the solution in the neighbourhood $\mathbb{R}^{n-2} \times \mathcal{U}$ of scri to all of $\mathbb{R}^{n-2} \times W \simeq \mathbb{R}^{n-2} \times D^{2}$.

Remark IV.2. One may impose in Theorem II1.2.1 and, subsequently, Theorem IV.1 a weaker regularity requirement, i.e., one may assume that $(\Sigma, h, N)$ is $C^{k}$ conformally compactifiable, for some $k \geq n+1$, but then the isometries constructed will in general be of finite differentiability.

Remark IV.3 (Non-Uniqueness). Let us consider, in Theorem IV.1, the somewhat more general situation in which $\partial \widetilde{\Sigma}$ is an $(n-1)$-torus with a fixed, but arbitrary, flat structure. Each free homotopy class of $\partial \widetilde{\Sigma}$ can be represented by a closed geodesic $\beta$. Suppose more generally, in Theorem IV.1, that a fixed but arbitrary embedded closed geodesic $\beta$ generates the kernel of $i_{*}$. One still has in this somewhat more general setting that $\pi_{1}(\widetilde{\Sigma})=\mathbb{Z}^{n-2}$. As in the proof of Theorem IV.1, it follows that the universal cover of $\widetilde{\Sigma}$ is isometric to Euclidean $(n-2)$-space times a 2-disk, whose boundary circle has length $L(\beta)=$ length of $\beta$. One can again solve for the geometry on the 2-disk to conclude that the universal cover of spacetime is isometric to an open adS soliton (spacetime with metric of the form I.1, but where the $\theta^{i}$ coordinates are no longer periodic but range through all of $\mathbb{R}$ ). Thus, the physical spacetime $M$ is isometric to an open adS soliton quotiented out by a certain $\mathbb{Z}^{n-2}$ action, $M \simeq$ (open AdS Soliton) $/ \mathbb{Z}^{n-2}$. Since the cycle $\beta$ may no longer be geometrically an $S^{1}$ factor of $\partial \widetilde{\Sigma}$, the $Z^{n-2}$-action may no longer fix the disk $W$, as it had done in part $(i)$ of the above proof, and we may lose the global geometric factorization (IV.11). Nonetheless, the relevant actions have been described explicitly in the $3+1$ (i.e., $n=3$ ) case in [2]. In this case one obtains a countable class of locally isometric but isometrically distinct spacetimes, generalized AdS solitons, whose mass depends on $L(\beta)$. Similar behavior will occur in higher dimensions.

Thus, fixing the conformal boundary, we obtain a spectrum of solitons of differing masses as we vary the choice of cycle $C$ representing ker $i_{*}$. For a given flat torus, the ground state soliton arises by choosing $C$ to be the shortest cycle on the torus. Other choices give rise to solitons that are excited states (they are still local minima of energy, by the Constable-Myers analysis [10]). The excited states have roles to play in the AdS/CFT correspondence. For example, they contribute to the Conformal Field Theory partition function [12. A particularly interesting application is to consider homotopically distinct closed geodesics $g_{1}, g_{2}, g_{3} \ldots$ on a given torus, ordered by increasing length. Consider the solitons $S_{1}, S_{2}$ that arise by taking $C_{1}$, respectively $C_{2}$, as the bulk-contractible cycle $C$. Then $S_{1}$ is the ground state and $S_{2}$ is the first excited state. Now vary the conformal structure so that the lengths of $g_{1}$ and $g_{2}$ cross, but remain less than the lengths of the other 
homotopically distinct cycles, so (the variation of) $S_{2}$ becomes the ground state. Page 32 has shown that this produces a zero temperature phase transition in the boundary CFT, as the pressures exerted on area elements transverse to $g_{1}$ and $g_{2}$ suddenly change sign.

\section{Concluding Remarks}

In a sequel to this paper [17, we make use of many of the techniques and results presented here to study static, asymptotically locally AdS spacetimes which contain black hole horizons (e.g., which contain non-naked singularities). For instance we show that a static spacetime asymptotic to the AdS soliton cannot have negative mass if it contains a horizon. This result is well illustrated by the toroidal Kottler spacetimes. In the sequel, we will also apply the results of Section II to study the case of an asymptotically locally AdS spacetime with conformal boundary admitting non-negative Ricci curvature.

\section{Acknowledgements}

This work was partially supported by grants from the National Science Foundation (USA) DMS-0104042 and the Natural Sciences and Engineering Research Council (Canada). SS was supported by a postdoctoral fellowship from the Pacific Institute for the Mathematical Sciences. GJG wishes to thank DAMTP, University of Cambridge, for hospitality during the completion of part of this work.

\section{Note Added}

After this paper was submitted, 3] appeared, containing related results in the 4-dimensional case.

\section{References}

[1] Abbott, L., and Deser, S., Nucl. Phys. B195, 2752 (1982).

[2] Anderson, M.T., Advances in Mathematics, to appear, math.DG/0104171.

[3] Anderson, M.T., Chruściel, P.T., and Delay, E., J High Energy Phys 10, 063 (2002) gr-qc/0211006.

[4] Ashtekar, A., and Magnon, A., Classical Quantum Gravity 1, L39 (1984).

[5] Boucher, W., Gibbons, G.W., and Horowitz, G.T., Phys. Rev. D30, 2447 (1984).

[6] Cadeau, C., and Woolgar, E., Classical Quantum Gravity 8, 527 (2001) gr-qc/0011029.

[7] Cheeger, J., and Gromoll, D., J. Differ. Geom. 6, 119 (1971); Ann. Math. 96, 413 (1972).

[8] Chruściel, P.T., and Herzlich, M., preprint (2001) math.DG/0110035.

[9] Chruściel, P.T., and Simon, W., J. Math. Phys. 42, 1779 (2001) gr-qc/0004032.

[10] Constable, N.R., and Myers, R.C., J. High Energy Phys. 9910, 037 (1999) hep-th/9908175. 
[11] de Haro, S., Skenderis, K., Solodukhin, S.N., Commun. Math. Phys. 217, 595 (2001) hep-th/0002230.

[12] Dijkgraaf, R., Maldacena, J., Moore, G., and Verlinde, E., preprint (2000) hep-th/0005003.

[13] Fefferman, C., and Graham, C.R., Astérisque, hors série, p. 95 (1985); Graham, C.R., Proc. 19th Winter School in Geometry and Physics, Srni, Czech Rep., Jan. 1999, math.DG/9909042.

[14] Galloway, G.J., Ann. Henri Poincaré 1, 543 (2000) math.DG/9909158.

[15] Galloway, G.J., Schleich, K., Witt, D.M., and Woolgar, E., Phys. Rev. D60, 104039 (1999) gr-qc/9902061.

[16] Galloway, G.J., Surya, S., and Woolgar, E., Phys. Rev. Lett. 88, 101102 (2002) hep-th/0108170.

[17] Galloway, G.J., Surya, S., and Woolgar, E., preprint (2002) gr-qc/0212079.

[18] Gao, S., and Wald, R.M., Classical Quantum Gravity 17, 4999 (2000) gr-qc/0007021.

[19] Gubser, S.S., Klebanov, I.R., and Polyakov, A.M., Phys. Lett. B428, 105 (1998) hep-th/9802109.

[20] Hawking, S.W. and Ellis, G.F.R., The large scale structure of space-time, Cambridge: Cambridge University Press, 1973.

[21] Hawking, S.W., and Horowitz, G.T., Classical Quantum Gravity 13, 1487 (1996).

[22] Hawking S.W., and Page, D.N., Commun. Math. Phys. 87, 577 (1983).

[23] Henneaux, M., and Teitelboim, C., Commun. Math. Phys. 98, 391 (1985).

[24] Henningson, M., and Skenderis, K., J. High Energy Phys. 9807, 023 (1998) hep-th/9806087.

[25] Horowitz, G.T., and Myers, R.C., Phys. Rev. D59, 026005 (1999) hep-th/9808079.

[26] Kiem, Y., and Park, D., Phys. Rev. D59, 044010 (1999) hep-th/9809174.

[27] Klebanov, I.R., in Quantum aspects of gauge theories, supersymmetry, and unification, Paris, 1-7 Sept 1999, available from J. High Energy Phys. Conference Archive PRHEP-tmr/99/026.

[28] Lemos, J.P.S., Phys. Lett. B352, 46 (1995).

[29] Lichnerowicz, A., C. R. Acad. Sci. 222, 432 (1946).

[30] Maldacena, J., Adv. Theor. Math. Phys. 2, 231 (1998) hep-th/9711200.

[31] Mann, R.B., in Internal Structure of Black Holes and Spacetime Singularities, eds. Burko, L., and Ori, A., Ann. Israeli Phys. Soc. 13, 311 (1998) gr-qc/9709039. 
[32] Page, D.N., preprint (2001) hep-th/0205001.

[33] Penrose, R., Sorkin, R.D., and Woolgar, E., preprint gr-qc/9301015.

[34] Petersen, P., Riemannian geometry, Graduate Texts in Mathematics, New York:Springer-Verlag, 1998.

[35] Schoen, R., and Yau, S.-T., Commun. Math. Phys. 79, 231 (1981).

[36] Susskind, L., J. Math. Phys. 36, 6377 (1995) hep-th/9409089.

[37] Surya, S., Schleich, K., and Witt, D.M., Phys. Rev. Lett. 86, 5231 (2001) hep-th/0101134.

[38] 't Hooft, G., in Salamfest, eds. Ali, A., Ellis, J., and Randjbar-Daemi, S., Singapore: World Scientific, 1994 gr-qc/9310026.

[39] Witten, E., Commun. Math. Phys. 80, 381 (1981).

[40] Witten, E., Adv. Theor. Math. Phys. 2, 253 (1998) hep-th/9802150.

[41] Woolgar, E., Classical Quantum Gravity 11, 1881 (1994) gr-qc/9404019. 


\section{A Hawking-Horowitz Mass}

Under the conformal transformation that replaces the geometry induced by the Fermat metric with that induced by the spacetime metric, the respective mean curvatures obey

$$
\begin{aligned}
H & =x \widetilde{H}-(n-1) \widetilde{n}^{c} \widetilde{\nabla}_{c} x \\
& =x \widetilde{H}+\frac{(n-1)}{\psi} .
\end{aligned}
$$

The $x \widetilde{H}$ term can be estimated using (ㅍ.2.19), but to estimate the second term requires some further work. We begin with the identity (II.1.7), which together with the definition $\widetilde{W}=1 / \psi^{2}$ gives

$$
\frac{1}{\psi^{2}}=\frac{-2 \Lambda-x^{2} \widetilde{R}}{n(n-1)}
$$

Now this can be estimated by combining (II.1.11), (II.2.21), and the estimates of Section II to obtain

$$
\widetilde{R}=\frac{x}{n-2} \frac{\partial \widetilde{R}}{\partial x}+\mathcal{O}\left(x^{n}\right)=\frac{x^{n-2}}{(n-2) !} \widetilde{R}^{(n-2)}(0)+\mathcal{O}\left(x^{n}\right),
$$

which can be substituted into (A.2) to yield

$$
\frac{1}{\psi^{2}}=\frac{-2 \Lambda}{n(n-1)}-\frac{x^{n}}{n !} \widetilde{R}^{(n-2)}(0)+\mathcal{O}\left(x^{n+2}\right) \quad .
$$

We take the square root, substitute the result into the last term in (A.1), and use (II.2.19) to estimate the first term, obtaining

$$
H=\frac{(n-1)}{\ell}+\frac{x^{n} \ell}{2(n !)} \widetilde{R}^{(n-2)}(0)+\mathcal{O}\left(x^{n+2}\right) .
$$

Following Hawking and Horowitz, we multiply $H$ by the lapse $N=1 / x$ and integrate over a constant $x$ surface $Y_{x}$ using the volume element $\sqrt{b}=\sqrt{\tilde{b}} / x^{n-1}$ of the (unrescaled) first fundamental form $b_{a b}:=N^{2} \widetilde{b}_{a b}$. This gives

$$
\int_{Y_{x}} N H \sqrt{b} d Y_{x}=\frac{(n-1)}{x^{n} \ell} \operatorname{vol}\left(Y_{x}\right)+\frac{\ell}{2(n !)} \int_{Y_{x}} \widetilde{R}^{(n-2)}(0) \sqrt{\widetilde{b}} d Y_{x}+\mathcal{O}\left(x^{2}\right)
$$

By the Hawking-Horowitz prescription, we must now embed a surface $Y_{x}^{\prime}$ in a slice of a reference spacetime, compute its mean curvature $H_{0}$, pull this back to $Y_{x}$ (call the pullback $H_{0}^{*}$ ), integrate it as above, compute the difference between the resulting integral and (A.6), and finally take $x \rightarrow 0$. The intrinsic geometry on $Y_{x}^{\prime}$ induced from the reference spacetime must match that on $Y_{x}$. The reference spacetime we will use is of constant negative curvature and is assumed to have the same Penrose conformal boundary as the physical spacetime (this may force the reference spacetime to be incomplete; see 25] for a discussion). We embed within a moment of time symmetry thereof, so the scalar curvature of this slice is constant. Thus

$$
H_{0}=\frac{(n-1)}{\ell}+\mathcal{O}\left(x^{n+2}\right)
$$


and thus

$$
\lim _{x \rightarrow 0} \int_{Y_{x}} N\left(H-H_{0}^{*}\right) \sqrt{b} d Y_{x}=\frac{\ell}{2(n !)} \int_{Y} \widetilde{R}^{(n-2)}(0) \sqrt{\widetilde{b}} d Y
$$

where $Y:=\lim _{x \rightarrow 0} Y_{x}$. Thus we have:

Proposition A.1. Let $(\Sigma, h, N)$ obey equations (II.1.2 II.1.3) and be $C^{n}$ conformally compactifiable. Then the Hawking-Horowitz mass $M_{H H}$ of $\Sigma$ is given by

$$
M_{H H}=\frac{-\ell}{16 \pi(n !)} \int_{Y} \widetilde{R}^{(n-2)}(0) \sqrt{\widetilde{b}} d Y
$$

Proof. Follows from (A.8) by comparing its left-hand side to the definition of $M_{H H}$ appearing in equation (2.14) of 21].

Remark A.2. By setting $Y=\partial \widetilde{\Sigma}$ and comparing (A.9) to (II.3.10), we have $M_{H H}=$ $M_{A M}$ in this setting.

\section{B Fermat and Null Lines}

Lemma B.1. Let $(\Sigma, h, N)$ be a static spacetime with lapse $N$ bounded from below. Let $\pi:\left(M, g_{a b}\right) \rightarrow\left(\widetilde{\Sigma}, \widetilde{h}_{a b}\right)$ be the projection along integral curves of the timelike Killing field $\partial / \partial t$. Then, for every Fermat line $\rho: \mathbb{R} \rightarrow\left(\widetilde{\Sigma}, \widetilde{h}_{a b}\right)$ through $p$ there is a unique future directed null line $\gamma: \mathbb{R} \rightarrow\left(M, g_{a b}\right)$ through $p$, with $\rho=\pi(\gamma)$.

Remark. There are in fact two null lines through $p$ that project to $\rho$, but one reverses orientation.

Proof. Let $\tilde{\sigma}$ be an arclength (i.e., affine) parameter along $\rho$. Without loss of generality, we can take $\rho$ to be of unit speed. On $\pi^{-1}(\rho)$, we can thus define the future directed null vector field, $\frac{\partial}{\partial t}+\dot{\rho}(\tilde{\sigma})$ and let $\gamma$ be the unique integral curve through $p$. Then along $\gamma$, we have $d t=d \tilde{\sigma}$.

We now show that $\gamma$ is achronal. Let us assume otherwise. Then there exist points $q, q^{\prime} \in \gamma$, with $q=\gamma(a), q^{\prime}=\gamma(b)$ and $a<b \in \mathbb{R}$, such that there is a timelike curve $\gamma^{\prime}$ from $q$ to $q^{\prime}$. Let $\rho^{\prime}$ be its projection into $\Sigma$. Since $d t=d \tilde{\sigma}$ along $\gamma$, we can integrate this to obtain $\Delta t:=b-a=\widetilde{L}(\rho)$, which is the Fermat length of $\rho$ between the projected points $\pi(q)$ and $\pi\left(q^{\prime}\right)$. Since $\rho$ is a Fermat line, $\widetilde{L}(\rho)$ equals the Fermat distance from $\pi(q)$ to $\pi\left(q^{\prime}\right)$. Using $\tilde{\sigma}$ to also parameterise $\gamma^{\prime}$, we see that $d t>d \tilde{\sigma}$, which integrates to give $\Delta t=b-a>\widetilde{L}\left(\rho^{\prime}\right)$, the length of $\rho^{\prime}$ between $\pi(q)$ and $\pi\left(q^{\prime}\right)$. But this means that $L\left(\rho^{\prime}\right)<L(\rho)$, which contradicts the claim that $\rho$ is minimal.

Finally, we show that $\gamma$ is also complete. That it is geodesic follows from the fact that it is null and achronal. However, the parameterisation $\tilde{\sigma}$ is not affine. Using the condition of staticity it can be shown that, up to a constant of proportionality, $\gamma$ has affine parameter $\lambda$ related to $\tilde{\sigma}$ by $d \lambda=N^{2} d t=N^{2} d \tilde{\sigma}$. Now, assume that $\gamma$ is incomplete, i.e., that it is inextendible in at least one direction in which it has a bounded range of affine parameter. Since $N$ is bounded away from zero, this means that the affine parameter $\tilde{\sigma}$ in that direction is also bounded above, so that $\rho$ would be incomplete, which again is a contradiction.

Thus, $\gamma$ is a complete achronal null line. 